\title{
Séchage d'un enduit pigmenté avec application à l'étude d'une couche papetière
}

\author{
P. Bernada et D. Bruneau (*) \\ L.E.P.T.-ENSAM, Esplanade des Arts et Métiers, 33405 Talence Cedex, France
}

(Reçu le 6 septembre 1995, revisé le 29 mars 1996, accepté le 12 avril 1996)

\author{
PACS.83.20.Hn - Structural and phase changes \\ PACS.83.70.Hq - Heterogeneous liquids: suspensions, dispersions, emulsions, pastes, slurries, \\ foams, block copolymers, etc. \\ PACS.47.55.Mh - Flows through porous media \\ PACS.44.90.+c - Other topics in heat transfer, thermal and thermodynamic processes
}

\begin{abstract}
Résumé. - Le séchage d'un enduit pigmenté à l'usage d'une couche papetière est abordé sous divers aspects : cinétiques de séchage et évolution temporelle des profils de teneur en eau, profils de répartition de la taille des pores dans la couche séchée, et répartition finale d'un liant hydrophile de la sauce de couchage (amidon). Les conséquences des conditions de séchage sur ces paramètres expérimentaux sont mises en évidence. De plus, les teneurs en eau caractéristiques de gélification et consolidation de la sauce de couchage sont obtenues par une approche graphique.
\end{abstract}

\begin{abstract}
Paper color coating drying is investigated from several experimental view points which include drying kinetics and time evolution of moisture content profiles, pore size distribution in the dried coating, and natural binder (starch) distribution in the dried coating. A study of the effects of drying conditions on those experimental parameters is carried out. Furthermore, gelification and consolidation characteristic moisture contents of the coating are determined using a graphical approach.
\end{abstract}

\section{Introduction}

Le procédé de fabrication du papier passe par plusieurs étapes : la pâte à papier, sous forme d'une suspension fibreuse, est déversée en continu sur une toile qui défile; elle s'y égoutte tout d'abord sous l'effet de dépressions successives produites par des éléments spécifiques d'égouttage. Le matelas fibreux ainsi obtenu est ensuite pressé, séché, calandré et expédié en imprimerie après bobinage.

Le produit obtenu peut être directement utilisé (impression d'un papier journal, par exemple). Il est peu onéreux mais possède une surface rugueuse et irrégulière constituée de fibres cellulosiques de différentes tailles enchevêtrées les unes aux autres.

Il existe un moyen d'améliorer l'imprimabilité du papier et de rendre sa surface très homogène. C'est de recouvrir sa surface d'un enduit, composé en majeure partie de charges minérales, de façon à boucher l'ensemble des irrégularités de cette surface. Cette opération d'enduction

$\left(^{*}\right)$ Auteur auquel doit être adressée la correspondance (e-mail: bruneau@lept-ensam.u-bordeaux.fr) 


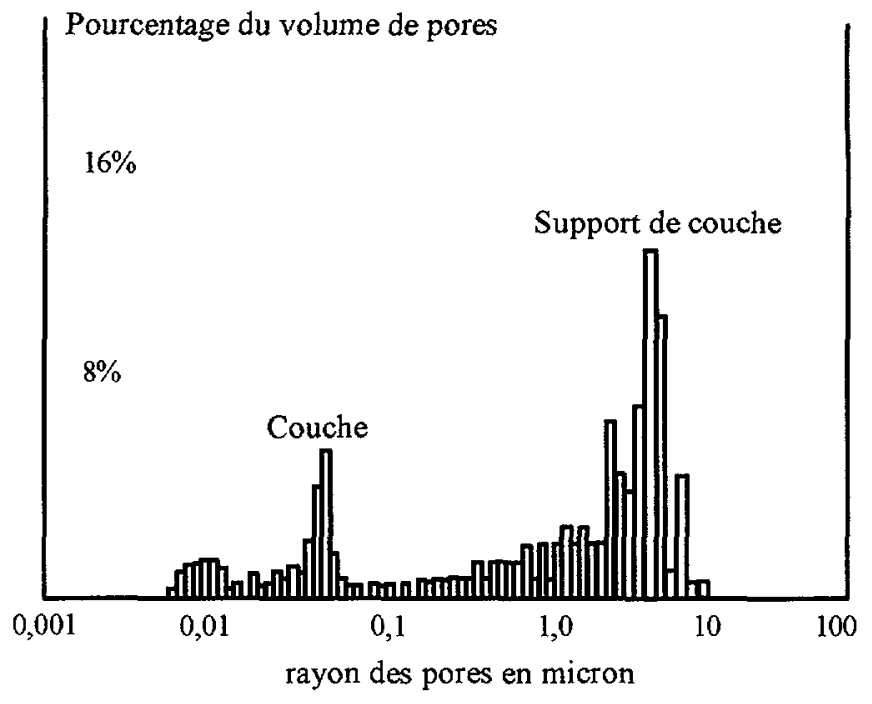

Fig. 1. - Distribution du rayon des pores dans une feuille de papier couché double face (d'après Nadeau et al. [2]).

[Pore size distribution in a dried two-sides coated paper (from Nadeau et al. [2]).]

d'un papier s'appelle couchage du papier. Le papier couché obtenu est un milieu poreux hétérogène, divisé en deux parties distinctes :

- la feuille de papier brute (appelée aussi papier support ou support de couche), dont l'épaisseur est de l'ordre de $100 \mu \mathrm{m}$, est un milieu poreux dont le rayon des pores est compris entre 1 et $10 \mu \mathrm{m}[1]$.

- L'enduit (appelé sauce de couchage) déposé sur le papier support forme une couche destinée à améliorer l'imprimabilité du papier ; l'épaisseur de cette couche varie entre 10 et $50 \mu \mathrm{m}$ suivant la fonction finale du produit. En fin de séchage, la couche sèche forme un milieu poreux consolidé dont le rayon des pores varie entre 0,01 et $0,1 \mu \mathrm{m}[2]$.

De façon générale, deux tailles distinctes de pores caractéristiques du papier couché peuvent être observées ; un exemple est donné pour un papier couché double face sur la figure 1.

Lors du passage dans la coucheuse-sécheuse industrielle, une certaine catégorie des constituants solides contenus dans la sauce (les liants) a une fầcheuse tendance à migrer vers la surface de la couche. Ces phénomènes de migrations des liants au cours du séchage entraînent des défauts dans le produit fini, défauts directement préjudiciables à une bonne qualité d'impression du papier (irrégularités d'impression, refus d'encre, etc...).

L'objet de cet article est une étude expérimentale de la migration d'un liant naturel, l'amidon, contenu dans une sauce de couchage lors du séchage de celle-ci. L'influence de l'intensité de séchage sur la porosité de surface des couches sèches obtenues est conjointement abordée. Dans ce but, nous présentons, dans un premier paragraphe, les différents phénomènes physiques qui interviennent au sein de la couche d'enduction lors du séchage de celle-ci. Le deuxième paragraphe est consacré à la description du système expérimental mis en œuvre pour sécher des couches de laboratoire, d'épaisseur initiale $1 \mathrm{~cm}$, ainsi qu'à l'interprétation des cinétiques de séchage et des évolutions temporelles des profils d'humidité obtenus. Les profils expérimentaux de porosité et de répartition d'amidon au sein de la couche permettent, dans les troisième 
et quatrième paragraphes, de mettre en évidence l'importance du début du séchage de la couche sur l'homogénéité finale de celle-ci. Enfin les conclusions sont énoncées dans le dernier paragraphe.

\section{Généralités sur les couches papetières}

1.1. Présentation de la SAUCe de Covchage. - On peut définir la couche par son but et sa composition.

Le but d'une couche papetière est d'embellir et d'améliorer certaines caractéristiques d'un papier, notamment la finition et l'imprimabilité du papier. La composition de la sauce de couchage comprend quatre éléments fondamentaux.

- L'eau : pour être appliquée sur le papier support brut, la sauce doit être suffisamment liquide. La quantité d'eau présente dans la sauce est mesurée par une grandeur appelée teneur en eau, dont la définition est la suivante : la teneur en eau d'un matériau est le rapport entre la masse d'eau qu'il contient $\left(m_{\mathrm{e}}\right)$ et une masse de référence qui peut être la masse totale de l'échantillon humide considéré ou la masse totale de l'échantillon sec $\left(m_{\mathrm{s}}\right)$. On parle alors de teneur en eau base humide ou humidité $\left(W_{\mathrm{b} . h .}\right.$. ) dans le premier cas et de teneur en eau base sèche ou humidité absolue $\left(W_{\mathrm{b} . s .}\right)$ dans le second :

$$
W_{\mathrm{b} . \mathrm{h} .}=\frac{m_{\mathrm{e}}}{m_{\mathrm{s}}+m_{\mathrm{e}}} \quad W_{\mathrm{b} . \mathrm{s} .}=\frac{m_{\mathrm{e}}}{m_{\mathrm{s}}}
$$

Pour des raisons de clarté de l'exposé, nous avons choisi de ne retenir qu'une seule de ces deux expressions. Dans tout ce qui suit, tous les résultats concernant les calculs ou les mesures de quantité d'eau dans la couche seront exprimés en terme de teneur en eau base sèche, que l'on notera $W$. Dans une sauce de couchage, la valeur de la teneur en eau initiale est de l'ordre de 0,54 .

- La charge, qui donne l'opacité et la blancheur à la couche. Elle constitue la structure même de la couche séchée et forme une surface poreuse mouillable à l'encre. Ce sont des minéraux naturels (kaolins, carbonates, etc.) ou synthétiques (dioxyde de titane) qui sont composés de particules insolubles dans l'eau ; leurs diamètres n'excèdent pas le micromètre. La taille et la forme de la charge, ainsi que sa masse volumique, vont influencer les transports des différents constituants solides et liquide qui prennent place lors du séchage de la couche.

- Un (ou plusieurs) adhésifs qu'on appelle aussi liants destinés à faire tenir la charge sur le support et à assurer la cohésion de la structure poreuse formée par la charge. Le but principal des liants, est de donner au papier couché une bonne tenue mécanique, tant au niveau de l'interface couche-papier, qu'au niveau de la cohésion mécanique des pigments entre eux. Cette tenue mécanique sera d'une importance capitale lors de l'impression de l'encre sur le papier, en raison des forces d'arrachage que celui-ci subit à ce moment là. Ces liants sont en général de deux types:

- Des liants naturels de type amidon. Du fait de sa structure chimique fortement hydrophile, l'amidon présente un comportement complexe intimement lié à sa teneur en eau. $\grave{A}$ des températures supérieures à $60^{\circ} \mathrm{C}$, une dispersion de grains d'amidon dans l'eau s'épaissit brusquement : l'eau chaude pénètre dans le grain d'amidon et le fait gonfler. On dit qu'il y a formation d'un empois d'amidon. Cet empois, en se refroidissant ou en perdant de l'eau, se gélifie. 
- Des liants synthétiques, de type latex qui sont des particules plastiques de dimension de l'ordre de $0,5 \mu \mathrm{m}$. Soumise à un flux d'évaporation, une solution d'eau et de latex va former un film à sa surface au cours du séchage (phénomène de coalescence des latex).

- Des additifs divers comme des dispersants, des modificateurs de viscosité, des antimousses, etc.

1.2. Comportement de la COUChe lors du séchage. - Lors de son passage dans la coucheuse, la structure intime de la sauce va subir plusieurs changements radicaux, changements induits par les opérations auxquelles la couche est soumise : l'enduction et le séchage en particulier, en induisant un flux d'eau et de liants respectivement vers le bas (flux d'imbibition) et le haut (flux de séchage) de la couche, vont modifier considérablement la structure interne de la sauce, amenant celle-ci progressivement de l'état liquide à celui d'un milieu poreux solide indéformable.

1.2.1. Évolution de la structure de la couche au cours du séchage. - En séchant la couche papetière perd peu à peu une partie de l'eau qu'elle contient et passe continûment par trois états successifs : état liquide, état de gel, état solide.

1.2.1.1. L'état liquide. - La sauce est alors un milieu diphasique composé d'un liquide visqueux dans lequel évoluent des constituants solides de différentes tailles et formes : des pigments, des liants qui peuvent être intimement liés à l'eau (amidon), ou non solubles dans l'eau (latex).

Lors du séchage d'une couche, le flux masse d'évaporation et le flux masse dû à la pénétration dans le papier support d'une partie de l'eau contenue dans la couche (imbibition), induisent une diminution de la teneur en eau du milieu ; les mouvements relatifs des constituants solides de la sauce sont de plus en plus gênés et ainsi, la viscosité de la sauce augmente.

1.2.1.2. L'état de gel. - Lorsque le séchage continue, la teneur en eau de la sauce diminue pour atteindre une valeur caractéristique $W_{\mathrm{g}}$, à partir de laquelle la sauce passe de l'état liquide désordonné à un état de gel partiellement ordonné. Cet ordonnancement s'effectue à la suite de la formation, par les liants, d'un réseau tridimensionnel déformable qui retient prisonnier les pigments. Il est à noter à ce niveau que tout les liants ne participent pas forcément dès le point de gel à la formation de cette structure de gel.

1.2.1.3. L'état solidifié. - La teneur en eau diminue encore au cours du séchage, pour atteindre une deuxième valeur caractéristique dite critique, notée $W_{c}$. Elle correspond au moment où le gel (structure tridimensionnelle déformable) se transforme en une structure solide indéformable. Les valeurs de la teneur en eau aux points de gel et critique dépendent principalement de la température locale de la sauce, du type et de la quantité de liants, de l'aptitude de ceux-ci à pouvoir se déformer.

1.2.2. Description succincte des différents modes de transport de l'eau et des liants. - La détermination des points de gel et de consolidation d'une sauce revêt un caractère important pour la prévision de la migration des liants ; en effet, Lepoutre et al. [3] suggèrent, avec l'appui d'expériences menées sur des couches comprenant des liants de types latex, que la migration de ceux-ci s'effectue par entraînement par la phase liquide, et ceci jusqu'au point de gel. De façon différente Vanderhoff [4], puis Lee et al. [5] proposent un mécanisme de transport des liants en tant que processus de filtration. Leur description n'exclue pas une migration des liants jusqu'au point de consolidation (où tous ceux-ci sont obligatoirement figés), si ces derniers sont de tailles inférieures au pores du milieu structuré qui se construit à partir du point de gel.

$\mathrm{Au}$ mode de transport classique de type convectif-diffusif, peut s'ajouter un processus de dissolution-déposition des liants. Ce processus suppose que les liants auxquels il s'applique sont 


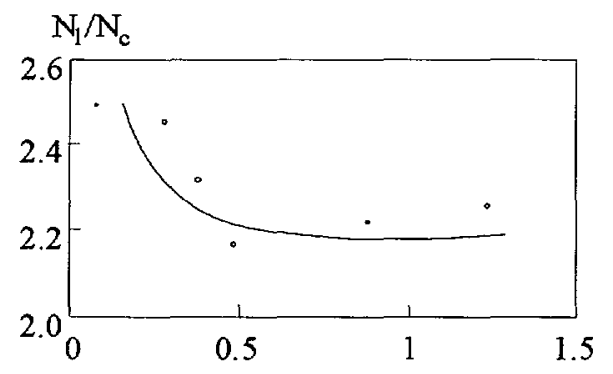

(s)

Fig. 2. - Influence de l'imbibition sur la migration des liants vers le haut de la couche; $N_{1} / N_{\mathrm{c}}$ représente ici le rapport entre les concentrations massiques de liants et de charges à la surface de la couche en fin de séchage (d'après Engström et al. [9]).

[Impregnation influence on binder migration to the top of the coating; $N_{1} / N_{c}$ is the ratio between binder mass concentration and pigment mass concentration at the top of the dried coating (from Engström et al. [9]).]

solubles dans la phase liquide. Au cours du séchage, la concentration massique locale en liants dissous augmente dans la phase liquide, pour atteindre finalement une valeur qui correspond à la saturation du liquide en liants. Dès que cette valeur de la concentration massique en liants est atteinte, les liants se solidifient et se déposent sur les constituants solides les plus proches. Ce mécanisme de dissolution-déposition a été utilisé par Farkas et al. [6], qui modélisent le latex comme un élément soluble dans la phase liquide.

Quel que soit le point d'arrêt des migrations, les différents auteurs sus-cités s'accordent pour affirmer que celles-ci s'effectuent conjointement avec le transport de la phase liquide.

Les sollicitations extérieures qui induisent ce mouvement fluide sont au nombre de deux : le séchage sur la face supérieure de la couche et l'effet d'imbibition de l'eau par le papier support. Ce processus d'imbibition est prépondérant juste après la phase d'enduction et devient négligeable au bout de quelques dixièmes de secondes [7]. Son effet est considérable sur la migration des liants dans la couche en déshydratant la partie de la couche située contre le papier support (base de la couche), l'imbibition aura pour effet d'amener cette partie de la sauce plus rapidement au point d'arrêt des migrations [5]. Il s'engage alors un phénomène physique irréversible : les liants qui ont migré dans le papier support au cours de l'imbibition, se retrouvent bloqués dans les macropores qui constituent la structure poreuse de ce support et ne peuvent plus remonter dans la couche [8]. Ce résultat est confirmé par Engström et al. [9] qui ont montré l'influence de la pénétration de l'eau dans le support sur le taux de liants en surface de la couche pour différentes vitesses de défilement du papier (Fig. 2).

Sur la figure 2, l'influence de la vitesse de défilement du papier est prise en compte par le temps d'arrivée au premier séchoir $\left(t_{1}\right)$ : plus ce temps est long, plus la vitesse de défilement est faible. On peut noter que le taux de liants à la surface supérieure de la couche est une fonction décroissante du temps d'arrivée au premier séchoir. Ce résultat peut s'expliquer de la manière suivante : lorsque $t_{1}$ est court, le flux d'adhésifs dirigé vers le bas de la couche et induit par le phénomène d'imbibition est tout de suite compensé par le flux de séchage dirigé vers le haut de la couche. Ainsi, une quantité importante de liants s'accumule à la surface de séchage. Lorsque $t_{1}$ est long, une quantité plus importante de liants est entraînée dans le papier support par effet d'imbibition, alors que du côté supérieur de la couche, le flux de séchage reste faible. Ainsi, une grande proportion de liants se retrouve immobilisée à la base de la couche. Le taux de liants à la surface supérieure en fin de séchage s'en trouve alors diminué. 
En ce qui concerne le transport de l'eau à l'intérieur de la couche, une majorité d'auteurs dans la littérature s'appuient sur ce modèle de transport capillaire pour des teneurs en eau supérieures à $W_{c}$ et même inférieures [1,7,8,10,11]. Seuls Nadeau et al. [2] et Lepoutre et al. [3] suggèrent que le transport de l'eau peut s'effectuer par diffusion vapeur ou écoulement de film pour des teneurs en eau locales inférieures à $W_{\mathrm{c}}$. Pour ces derniers auteurs, la sauce se contracte d'un volume égal à sa perte en eau locale jusqu'au point critique (retrait idéal). Au delà, la sauce est un solide indéformable et l'eau évacuée est remplacée par une phase gazeuse. Ainsi, dès que la surface de la couche atteint le point critique, le front de séchage (d'équation $W=W_{\mathrm{c}}$ ) pénètre dans celle-ci.

Vanderhoff et al. [12] semblent se démarquer de l'idée générale suivant laquelle le transport de la phase liquide par capillarité est un facteur prépondérant du transport de l'eau dans la couche (et de la migration des liants par entraînement ou processus de dissolution-déposition). Les raisons avancées sont les suivantes :

1) Ces auteurs ont montré à partir d'un modèle expérimental que la distribution des liants à la surface de la couche ne dépend pas de la taille des pores autour desquels ils se sont déposés. Le transport par capillarité indique quant à lui que c'est surtout dans les pores de grandes dimensions qu'il y aurait le plus de migration et donc de déposition de liants. Ces deux observations les amènent à dire que le transport fluide prédominant ne s'effectue pas par capillarité.

2) De plus, pour ces deux auteurs, le transport par capillarité ne peut avoir lieu que quand la structure solide de la couche est rigide et indéformable (i.e. après le point critique). Or, à ce moment là du séchage, les liants font partie intégrante de la structure solide et leur migration est impossible. Ainsi, d'après ces auteurs, le transport capillaire qui pourrait éventuellement prendre place à la fin du séchage de la couche, ne suffit pas à expliquer la migrations des liants.

En ce qui concerne l'éventualité d'un transport capillaire, les travaux de Moyne [13] et Couture [14] sur le séchage des matériaux capillaro-poreux, montrent que l'écoulement par capillarité n'est concevable que si le rayon du pore dans lequel il s'est établi est an minimum de l'ordre de grandeur du micron. En effet, les molécules d'eau situées à une distance inférieure à $1 \mu \mathrm{m}$ d'une paroi, sont intimement liées à celle-ci du fait de I'importance des forces de Van der Waals et électrostatiques dans cette région. L'énergie nécessaire pour arracher ces molécules d'eau de la matrice solide et les mettre en mouvement, devient non négligeable devant la chaleur latente de vaporisation de l'eau. Ainsi, dans un pore de rayon inférieur au micron, la pression capillaire ne suffit pas à créer l'écoulement.

Les résultats reportés sur la figure 1 montrent que les pores de la couche ont un rayon inférieur à $0.1 \mu \mathrm{m}$. D'après les remarques précédentes faites sur le lien entre l'écoulement d'un liquide dans un pore par capillarité et le rayon de ce pore, on peut donc considérer que lorsque la couche a atteint le point critique, l'écoulement par capillarité est impossible. D'autre part, le processus de transport capillaire pour ces teneurs en eau supérieures à $W_{\mathrm{c}}$ nous paraît peu fiable. En effet, pour un milieu liquide ou à l'état de gel le concept de perméabilité à l'eau ne peut être justifié.

En conclusion de cette rapide revue bibliographique, nous retiendrons les points suivants :

- Les transferts d'eau au sein de la couche se font sous forme liquide pour des teneurs en eau locales supérieures à $W_{c}$; le retrait local est alors idéal (pas de phase gazeuse). Pour des teneurs en eau locales inférieures à $W_{c}$, les transferts d'eau se font sous forme de film liquide ou par diffusion vapeur; le retrait est alors nul. Le front de séchage se déplace vers l'intérieur de la couche au rythme de la frontière $W=W_{\mathrm{c}}$.

- La migration des liants s'effectue au moins jusqu'au point de gel. Elle peut se prolonger pour une partie de ceux-ci jusqu'au point critique. 


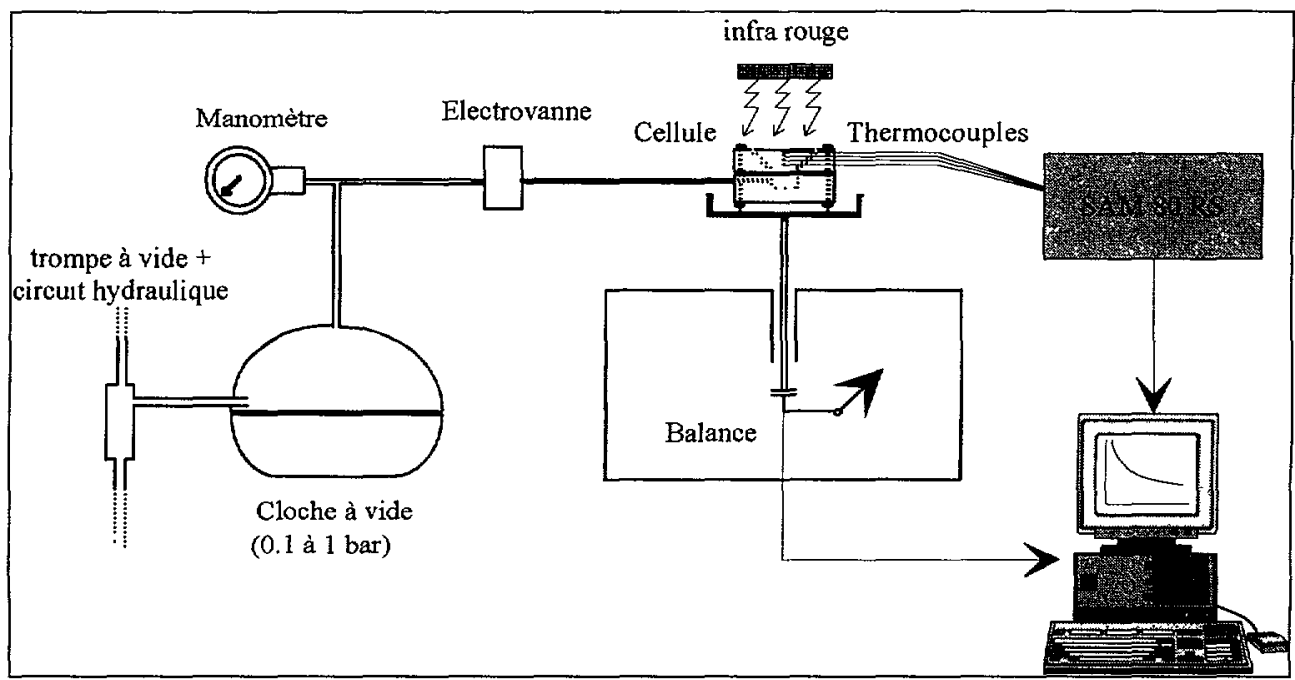

Fig. 3. - Représentation schématique du montage expérimental.

[Experimental configuration.]

2. Analyse en terme de séchage : détermination des teneurs en eau de gel et de consolidation de la sauce

Ce paragraphe est consacré à une analyse des couches papetières en terme de séchage. La détermination des cinétiques de séchage et d'imbibition et des profils de teneurs en eau amène à une étude fine du couplage entre les différentes phases de séchage et la consolidation de la couche. Les teneurs en eau caractéristiques de changement d'état de la structure de la couche, i.e. la teneur en eau au point de gel $W_{\mathrm{g}}$ et la teneur en eau au point critique $W_{\mathrm{c}}$, ont pu être mesurées par méthode graphique. Certains des résultats obtenus sur les couches papetières sont comparés à ceux obtenus sur des couches plus simples composées uniquement de kaolin, ceci de manière à déterminer l'influence de la présence des liants sur le transport liquide dans la couche.

2.1. Présentation du système expérimental de séchage. - On a vu précédemment qu'en situation industrielle, l'imbibition de l'eau dans le papier support était prépondérante dans les premiers instants du séchage et qu'une quantité non négligeable de l'eau contenue dans la couche était ainsi absorbée par le support. L'idée de départ a donc été de concevoir un système expérimental qui permet à la fois de sécher la couche par le dessus et de "simuler" ce processus d'imbibition. Ce système est décrit sur la figure 3 ; il comporte

- un dessicateur à températures réglables fonctionnant à l'aide de deux émetteurs infrarouge moyen,

- une cellule de mesure munie d'un fond poreux permettant, par aspiration, de simuler l'imbibition de l'eau de la couche par le support papier. Cette cellule qui contient les couches à sécher est représentée sur la figure 4 ,

- une cloche à vide qui, reliée à une trompe à vide, permet de descendre à des pressions de l'ordre de 0,05 bar, 


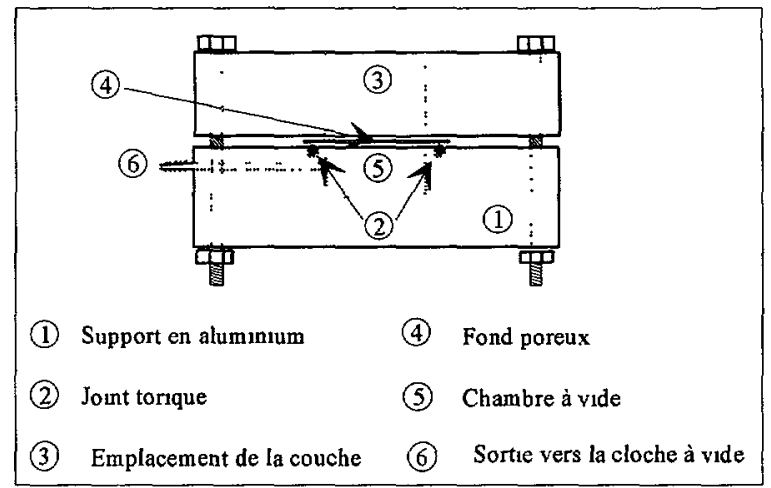

Fig. 4. - Schéma de la cellule employée pour les expériences de séchage de couche de laboratoire. [Schematic of the experimental cell used for the laboratory coating drying experiments.]

- une électrovanne qui, placée entre la cloche à vide et la cellule, permet de déclencher ou non l'imbibition sous la couche,

- une balance précise à $10^{-3}$ gramme près qui donne l'évolution de la masse de la cellule au cours du séchage,

- un logiciel d'acquisition : relié à la balance par l'intermédiaire d'une liaison RS232, il permet l'acquisition de la masse globale au cours du temps.

Le protocole expérimental réalisé lors d'une simulation expérimentale d'imbibition peut être décomposé comme suit :

1- Ouverture de la vanne d'entrée de la cloche à vide. L'électrovanne doit être en position fermée, lors de la mise sous vide de la cloche.

2- Ouverture du circuit hydraulique : l'air présent dans la cloche à vide est en partie aspirée au niveau de la trompe à vide et la pression chute dans la cloche. Des courbes de pression en fonction du temps sont représentées sur la figure 5. Pour deux essais différents, les courbes sont quasiment identiques et la répétitivité de la manipulation est assurée. Au bout de 20 minutes la pression dans l'enceinte se stabilise à la valeur de 0,05 bar.

3- Une fois la valeur limite de la pression dans la cloche à vide atteinte, on envoie un signal électrique à l'électrovanne qui ouvre le circuit d'accès à la cellule de mesure. En quelques dixièmes de seconde, le faible volume d'air contenu dans la chambre à vide et le tube situé en aval de l'électrovanne, est évacué vers la cloche. De ce fait, la pression interne de celle-ci remonte légèrement pour se stabiliser à une valeur de 0,1 bar.

Pendant la phase de simulation de l'imbibition, les transferts de masse entre le bas de la couche et la chambre à vide qui se font à travers la membrane poreuse, sont considérables du fait des faibles pressions qui règnent dans la chambre à vide. Il est même possible que lors d'expériences menées à des températures relativement élevées, la pression de vapeur de l'enceinte atteigne la pression de vapeur saturante et qu'un phénomène de vaporisation de l'eau se développe à la frontière inférieure de la couche pendant les premières minutes de l'expérience. Le flux masse d'évaporation induit par cette chute de pression dans l'enceinte de la cellule est nommé par la suite "flux d'imbibition". 


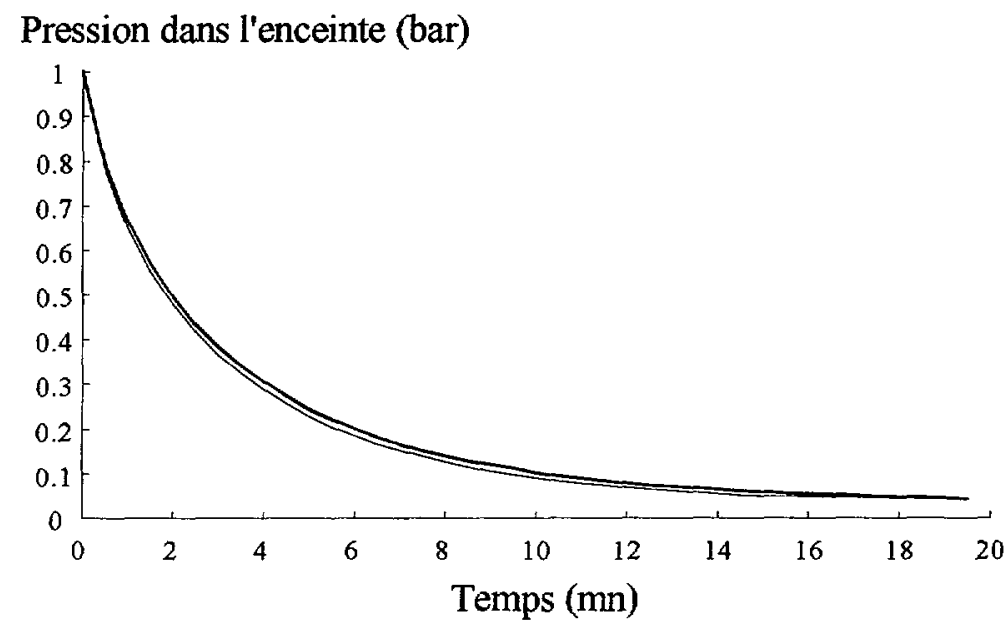

Fig. 5. - Évolution de la pression dans la cloche à vide au cours du temps ; visualisation de la répétitivité des vides imposés.

[Pressure evolution in the vacuum chamber during time; visualization of imposed vacuum repetitivity.]

4- Arrêt de la phase de vide active par fermeture du circuit hydraulique et fermeture de l'électrovanne. Du fait d'un léger débit de fuite intervenant au niveau de la liaison entre la cellule de mesure et le tuyau qui relie celle-ci à l'électrovanne, la pression sous le support poreux remonte naturellement en environ $15 \mathrm{~min}$. Dans tous les cas d'expériences réalisées ici, la durée de la phase active de vide a été fixée à 1 heure, ce qui permet d'obtenir une perte d'eau par simulation d'imbibition non négligeable au regard de celle provenant du séchage (voir Fig. 8).

Volontairement, l'échelle des essais (couches d'épaisseurs de $1 \mathrm{~cm}$ ) est très largement supérieure à celle rencontrée sur les coucheuses industrielles (couches d'épaisseur maximale $50 \mu \mathrm{m}$ ) et ce pour une raison simple : un des buts de l'étude expérimentale est la détermination des profils de teneur en eau et de concentration de liant (ici l'amidon) dans l'épaisseur de la couche. En ce qui concerne la répartition en liants en fin de séchage, nous pouvons citer les travaux de Mailly Lejeune [15], qui par une technique d'abrasion a pu déterminer la répartition d'un liants de type latex dans des couches de 6 à $14 \mu \mathrm{m}$. Par contre, il est à noter qu'il n'existe actuellement pas de techniques expérimentales permettent de déterminer en continu des profils de teneur en eau au cours du séchage de couches de telles épaisseurs : les techniques actuellement en place amènent uniquement à mesurer les humidités correspondant au point de gel et point critique de la sauce considérée [3,16]. Ainsi, afin d'obtenir ces profils par une méthode de découpe destructive, nous avons choisi d'étudier des couches épaisses qui diffèrent assurément d'une couche papetière réelle.

Avant de passer aux résultats des manipulations, il convient de noter la composition des sauces de couchage utilisées lors des essais. Afin de caractériser l'influence des liants sur les cinétiques globales de teneur en eau, sur les propriétés de transport liquide dans la couche ainsi que sur la porosité finale de la couche séchée, deux couches de référence ont été testées :

- La couche de base composée uniquement d'eau et de kaolin (plaquettes d'argiles de dimension caractéristique de l'ordre du micron). Les proportions massiques des deux éléments sont : $65 \%$ de kaolin et $35 \%$ d'eau. 
- La couche papetière (complète) dont la composition est la suivante (proportions massiques) :

- $57,5 \%$ de matière minérale (kaolin + carbonate),

- $35 \%$ d'eau,

- $5,75 \%$ de latex,

$-1,75 \%$ d'amidon.

2.2. Présentation des cinétiques de séchage et interprétation. - Ce paragraphe est consacré dans un premier temps à l'analyse du séchage de la couche, sans simulation d'imbibition et à l'interprétation des cinétiques de masse en terme de flux de séchage. Dans un deuxième temps, le flux d'imbibition créé lors de la mise sous vide partiel du fond de la couche est mis en évidence.

2.2.1. Séchage d'une couche sans simulation d'imbibition. Analyse des cinétiques de séchage des deux couches de référence. - Les résultats exprimés en terme de cinétique de séchage sont reportés sur la figure $6 \mathrm{a}$. L'influence de la température de séchage sur la cinétique de séchage apparaît clairement. D'autre part, pour une température de séchage donnée, la couche de kaolin sèche plus vite qu'une couche papetière. La principale différence de composition des deux types de couche est que la couche papetière contient des liants (latex et amidon) alors que l'autre n'est composée que d'eau et de matières minérales (kaolin). Ainsi, les résultats mettent en évidence un effet, provoqué par les liants, de ralentissement sur le séchage de la couche.

L'analyse des figures $6 \mathrm{~b}$ et $6 \mathrm{c}$, qui représentent l'évolution du flux masse d'eau s'évacuant de la couche (masse d'eau au cours du temps) en fonction de la teneur en eau base sèche moyenne de la couche, notée ici $W_{\text {glo }}$, confirme la remarque précédente et nous permet d'étoffer notre interprétation :

- Dans le cas d'une couche composée d'eau et de kaolin : les deux phases classiques de séchage d'un matériau non hygroscopique sont ici mises en évidence :

1- La phase isenthalpe, durant laquelle l'eau est évacuée de la couche à flux masse constant. L'évaporation s'effectue à la surface du matériau. Cette période existe tant que la surface du matériau est suffisamment alimentée en eau.

2- La première période de ralentissement qui commence lorsque la teneur en eau globale de la couche passe en deçà de 0,3 environ. À partir de cet instant, la zone d'évaporation qui se trouvait en surface se déplace vers l'intérieur du matériau. La sauce située au dessus du front de séchage est consolidée. La brusque réduction de la surface effective de transfert due à une alimentation insuffisante en eau de la surface de la couche est la cause de ce ralentissement. Alors qu'en phase isenthalpe c'est l'environnement séchant qui impose l'allure de séchage, dans cette période ce sont les diverses résistances aux transferts de la couche entre le front de séchage et la surface de celle-ci qui gèrent l'allure de la cinétique.

Il est à noter que l'on ne distingue pas nettement de troisième phase (classique) de séchage caractérisée par une nouvelle rupture de pente dans le diagramme $F_{\mathrm{m}}\left(W_{\mathrm{glo}}\right)$; cette absence de diminution brutale du flux masse en fin de séchage nous permet d'affirmer que le kaolin n'est pas un matériau hygroscopique : c'est en effet un matériau sans affinité chimique avec l'eau. 


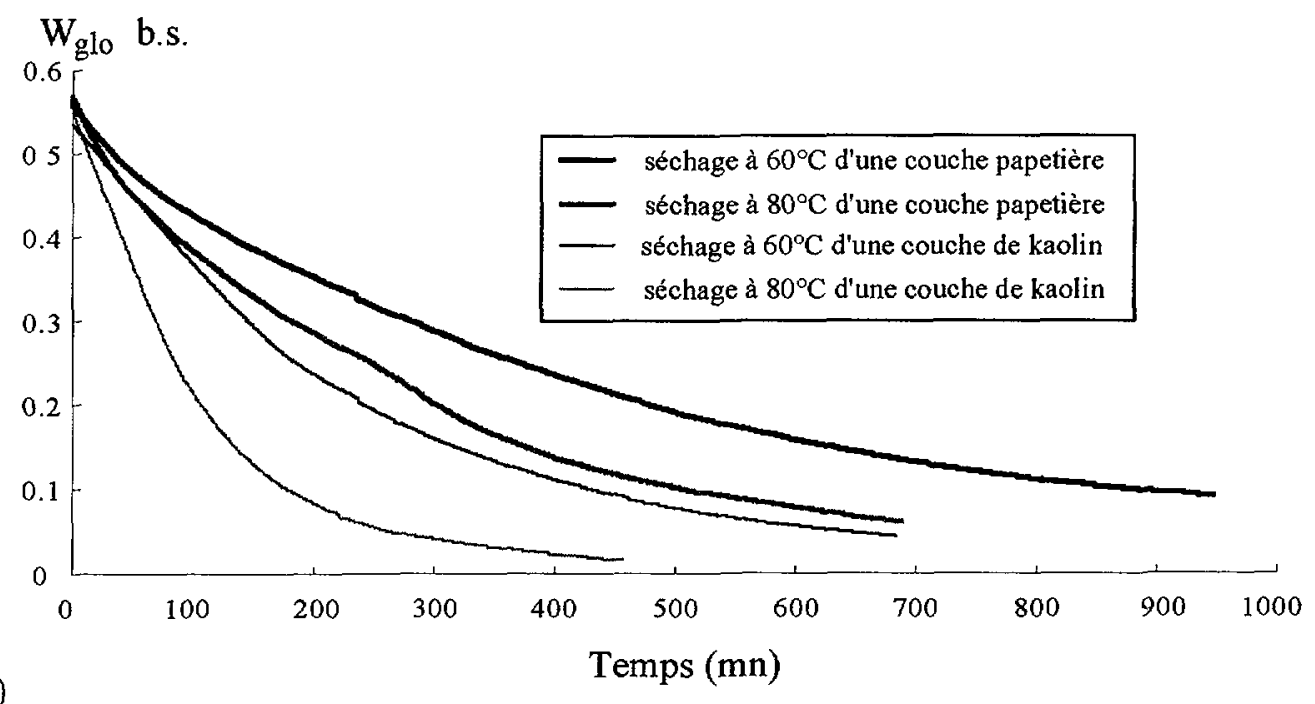

a)

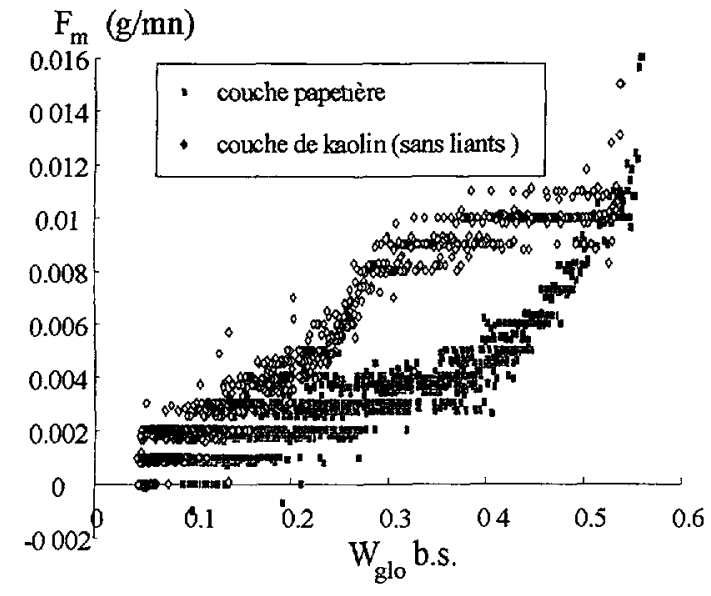

b)

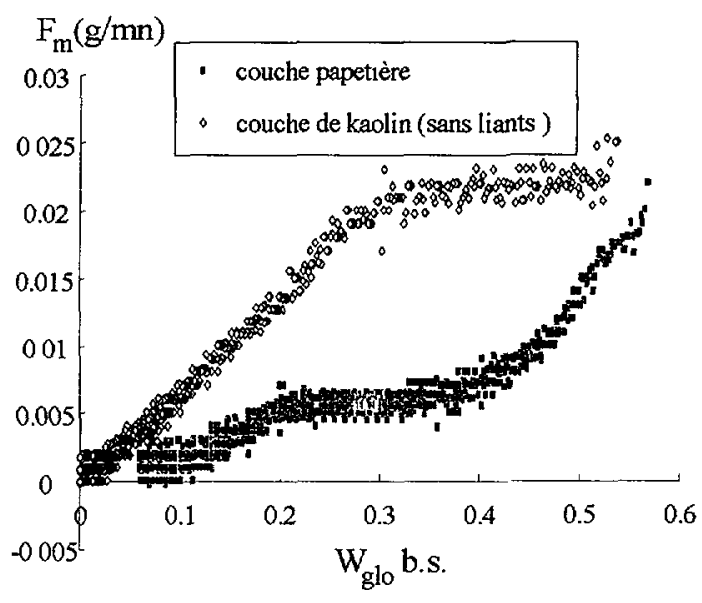

c)

Fig. 6. - Représentation de cinétiques de séchage. Influence de la température et de la composition de la sauce ( $W_{\text {glo }}$ est la teneur en eau globale moyenne de la couche). a) Évolution de la teneur en eau globale au cours du temps : comparaison des cinétiques pour deux compositions de couche différentes et deux températures de séchage. b) Évolution du flux masse d'eau évaporée en fonction de la teneur en eau globale pour un séchage à $60^{\circ} \mathrm{C}$ sans simulation d'imbibition. c) Évolution du flux masse d'eau évaporée en fonction de la teneur en eau globale pour un séchage à $80^{\circ} \mathrm{C}$ sans simulation d'imbibition.

[On the drying kinetics. The influence of temperature and coating composition ( $W_{\text {glo }}$ is the global coating moisture content). a) Global moisture content evolution during time: kinetics comparison for two coating compositions and two drying temperatures. b) Mass flux evolution of evaporated water vs. global moisture content for a $60^{\circ} \mathrm{C}$ drying experiment without any impregnation simulation. c) Mass flux evolution of evaporated water vs. global moisture content for an $80^{\circ} \mathrm{C}$ drying experiment without any impregnation simulation.] 
- Dans le cas du séchage d'une couche papetière : on distingue nettement trois périodes de séchage :

1- Une phase durant laquelle le flux masse d'eau évaporée chute. Cette phase se termine Iorsque la teneur en eau globale de la couche atteint la valeur 0,32 (environ).

2 - Une phase quasi-isenthalpe $\left(W_{\text {glo }} \in[0,23 ; 0,32]\right)$ durant laquelle l'eau est évaporée à flux masse constant.

3- Une deuxième phase de ralentissement $\left(W_{\text {glo }} \in[0 ; 0,23]\right)$ pour laquelle on observe une nouvelle chute du flux masse.

L'apparition prématurée d'une phase de ralentissement pour des teneurs en eau relativement élevées dans la cas du séchage d'une couche papetière a retenu toute notre attention de par son caractère non classique. Elle peut s'expliquer par la présence des liants dans la sauce : dès le début du séchage, les liants coalescent à la surface de la couche et un film fin se constitue. Un bilan d'énergie sur la couche amène à remarquer que l'énergie fournie à la couche par unité de temps $\left(E_{\mathrm{f}}\right)$ sert à la fois à l'évaporation de l'eau $\left(E_{\text {év }}\right)$ et à la consolidation de la structure $\left(E_{\mathrm{c}}\right)$ :

$$
E_{\mathrm{f}}=E_{\mathrm{év}}+E_{\mathrm{c}}
$$

Dans l'équation précédente, $E_{\text {év }}$ est liée au flux masse par la relation $E_{\text {év }}=F_{\mathrm{m}} \Delta H$ où $\Delta H$ est la chaleur latente de vaporisation de l'eau.

Dans le cas du séchage d'une couche de kaolin, l'énergie nécessaire à la consolidation de l'édifice est négligeable dans les premiers instants du séchage. Ceci peut s'exprimer comme suit :

$$
F_{\mathrm{m}}=\frac{E_{\mathrm{f}}}{\Delta H}
$$

Lors du séchage d'une couche papetière (kaolin + carbonates + liants), l'énergie consommée lors de la coalescence des liants n'est plus négligeable :

$$
F_{\mathrm{m}}=\frac{E_{\mathrm{f}}-E_{\mathrm{c}}}{\Delta H}
$$

Ainsi, pour une même énergie fournie à la couche, le flux masse est plus faible dans le cas du séchage d'une couche papetière que pour une simple couche de kaolin.

Pendant cette première phase de séchage, le film de liants formé à la surface de la couche papetière s'épaissit pour finalement gagner progressivement toute la couche pour une teneur en eau globale de celle-ci $W_{\text {glo }} \approx 0,32$; le phénomène de coalescence des liants est alors terminé et le flux masse se stabilise à une valeur environ trois fois plus faible que sa valeur initiale. Démarre alors une phase quasi-isenthalpe classique qui s'interrompt à une teneur en eau globale égale environ à 0,23 . Cette dernière valeur de teneur en eau globale qui correspond au début de la phase de ralentissement (pénétration du front de séchage dans la couche) est inférieure à celle observée pour une couche de kaolin pure ; cet état de fait peut d'ores et déjà laisser penser que la teneur en eau au point critique de la sauce complète est probablement inférieure à celle de la sauce constituée de kaolin et d'eau : en effet, à l'instant où le front de séchage pénètre dans le matériau, la teneur en eau locale au sommet de celui-ci est celle du point critique.

Les trois phases de séchage de la sauce complète et les remarques qui y sont assujetties sont résumées dans la figure 7 . 


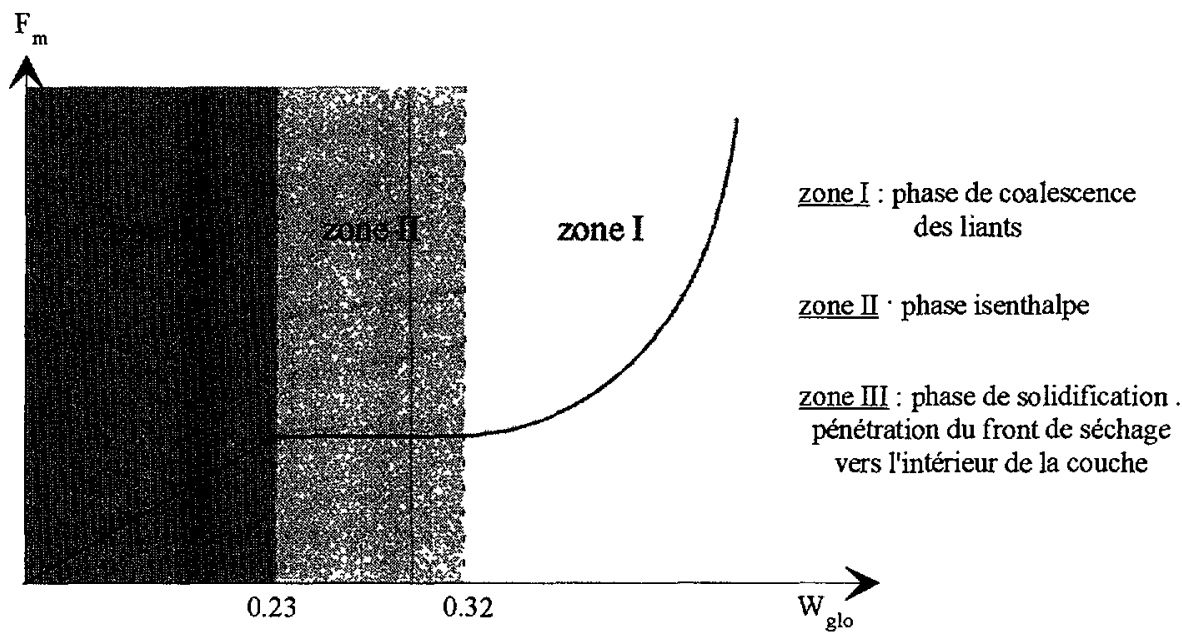

Fig. 7. - Séchage d'une couche papetière; présentation des trois phases caractéristiques.

[Paper coating drying; characteristic steps presentation. ]

2.2.2. Validation de la simulation expérimentale de l'imbibition. - Des cinétiques de séchage à $60^{\circ} \mathrm{C}$ et $80^{\circ} \mathrm{C}$, avec ou sans simulation d'imbibition, pour une sauce de couchage sont représentées sur la figure $8 \mathrm{a}$.

Il est à noter que les deux cinétiques de séchage avec simulation de l'imbibition sont beaucoup plus "accidentées" que les cinétiques de séchage pur. Ce phénomène provient simplement de l'influence du montage expérimental sur la mesure de la masse de la cellule. En effet, l'ordre de grandeur des flux mesurés est d'environ $0,05 \mathrm{~g} \mathrm{~min}^{-1}$ Dans le cas où l'imbibition est simulée, la cellule est reliée à la cloche à vide par l'intermédiaire d'un tuyau en matière plastique. La moindre action agissant sur l'un de ces trois éléments est directement préjudiciable à la qualité de la mesure de la masse de la couche. Malgré toutes les précautions prises, certaines manipulations nécessaires aux expériences n'ont pu être évitées : en particulier les chocs créés lors de l'ouverture et la fermeture de l'électrovanne entraîne des mouvements ou déformations du tuyau reliant l'électrovanne à la cellule de mesure. Ces déformations, même infimes, engendrent à leur tour des efforts qui sont enregistrés par la balance et ainsi nuisent à la précision de la mesure.

La première série de courbes (Fig. 8a) nous permet de valider le montage expérimental qui a été conçu pour simuler l'imbibition : les pertes en masse des couches sont plus rapides quand l'imbibition est simulée sous la cellule de mesure. Le fait d'imposer un vide relatif relativement poussé dans la chambre à vide a bien créé un flux d'évaporation non négligeable à la frontière inférieure de la couche.

Les figures $8 \mathrm{~b}$ et $8 \mathrm{c}$ font chacune apparaître trois courbes :

- le flux masse total en présence d'imbibition ; celui-ci est obtenu directement en pesant la couche au cours du temps,

- le flux masse de séchage ; celui-ci est obtenu par pesée, pour une manipulation de séchage sans simulation d'imbibition effectuée à la même température.

- le flux masse d'imbibition ; ce dernier flux est la différence entre les deux flux sus-cités. 


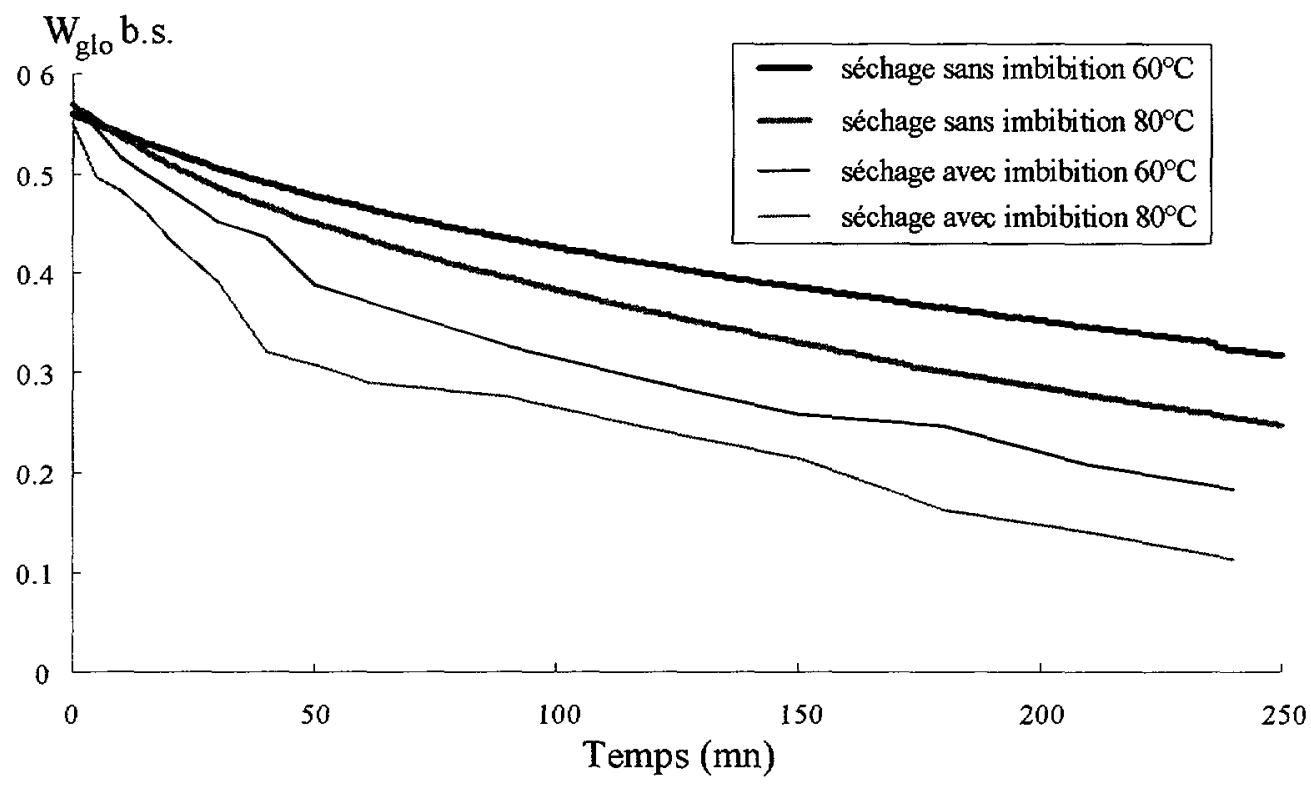

a)

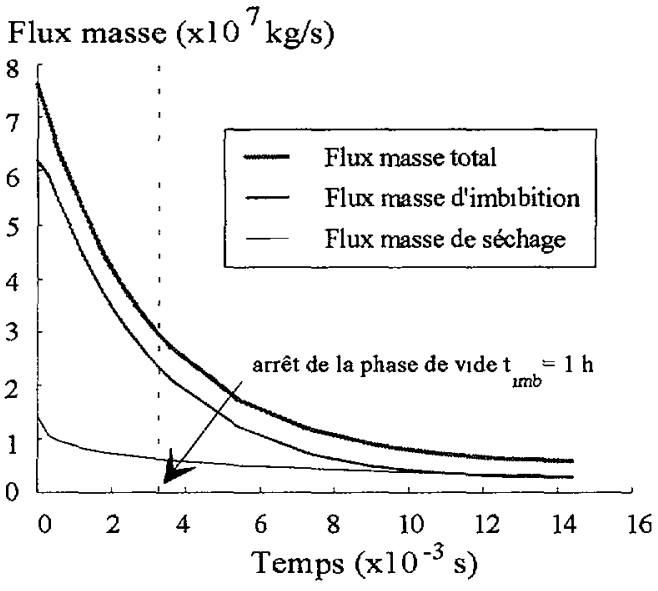

b)

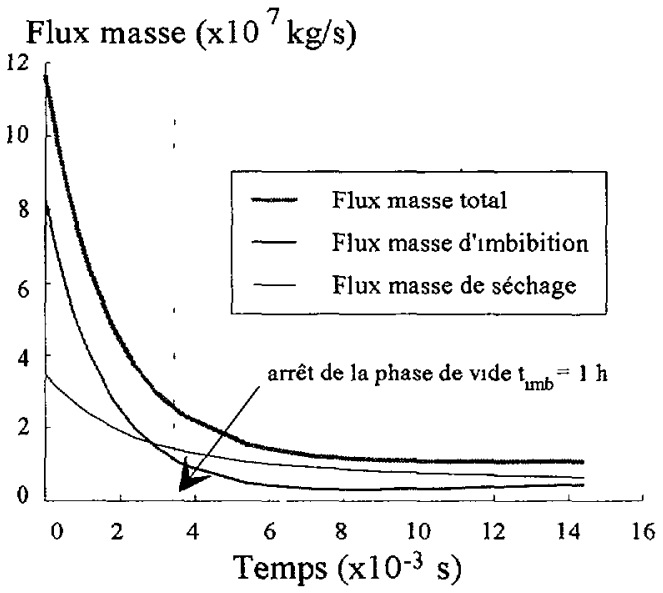

c)

Fig. 8. - Représentation de cinétiques de séchage. Validation de la simulation de l'imbibition ; influence de la température de séchage sur celle-ci. a) Évolution de la teneur en eau globale au cours du temps. b) Évolution du flux masse au cours du temps pour une température de séchage de $60{ }^{\circ} \mathrm{C}$ : comparaison du flux total, du flux d'imbibition et du flux de séchage. c) Évolution du flux masse au cours du temps pour une température de séchage de $80^{\circ} \mathrm{C}$ : comparaison du flux total, du flux d'imbibition et du flux de séchage.

[On the drying kinetics. Impregnation simulation validation and influence of drying temperature on the impregnation simulation. a) Global moisture content evolution during time. b) Mass flux evolution during time for a $60{ }^{\circ} \mathrm{C}$ drying experiment: comparison of the total mass flux, the impregnation mass flux and the drying mass flux. c) Mass flux evolution during time for an $80^{\circ} \mathrm{C}$ drying experiment: comparison of the total mass flux, the impregnation mass flux and the drying mass flux.] 


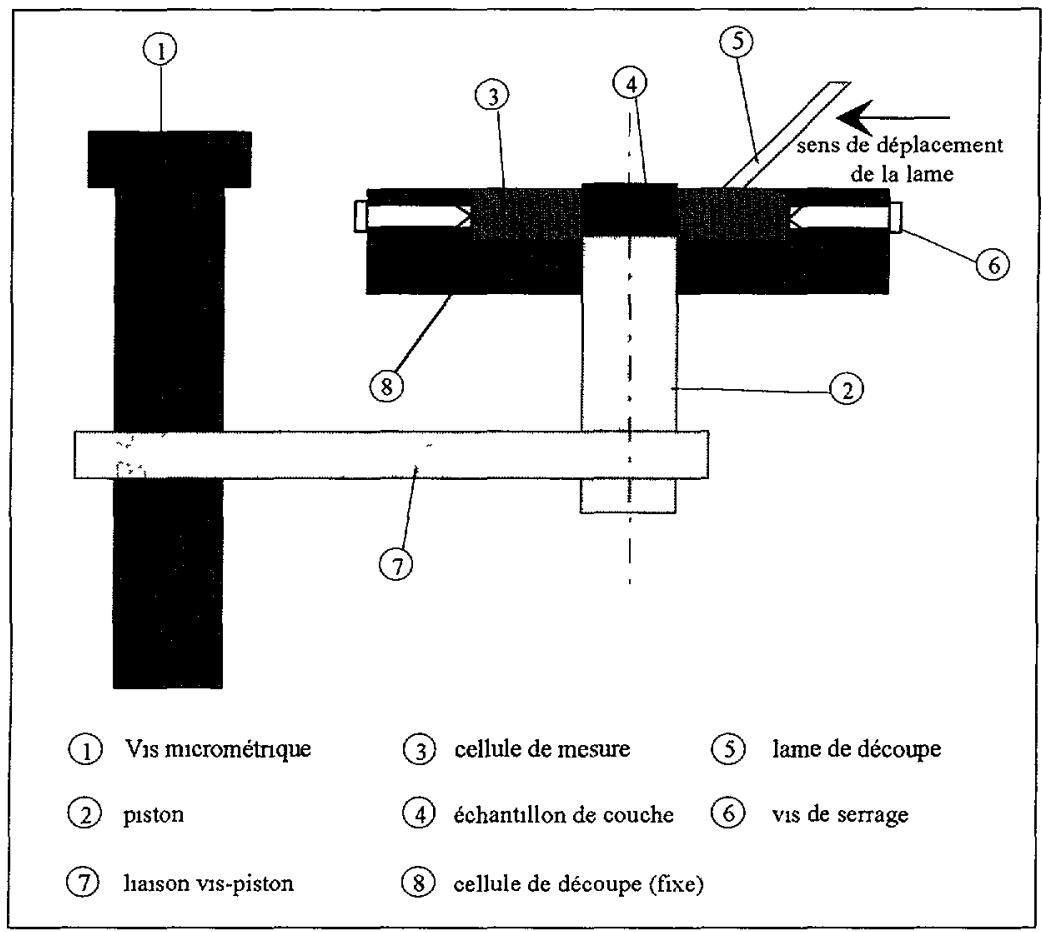

Fig. 9. - Schéma du dispositif de découpe d'une couche papetière.

[Coating cutting configuration.]

Il peut être noté que le flux masse d'imbibition est plus important quand la température de séchage est élevée; une explication peut être recherchée du côté de l'évolution de la viscosité de la sauce en fonction de sa température : celle-ci est une fonction décroissante ; ainsi, pour une dépression fixée en dessous de la couche, l'eau s'évacue plus facilement de celle-ci pour un séchage à $80^{\circ} \mathrm{C}$ car elle est moins gênée par les constituants solides de la sauce de couchage.

\subsection{Obtention de l'Évolution temporelle des profils de teneur en eau. DÉTERMINATION DES POINTS DE GEL ET CRITIQUE}

2.3.1. Le montage expérimental. - Afin de caractériser le transport interne de la phase liquide au cours du séchage, un procédé expérimental permettant la détermination des profils de teneur en eau dans l'épaisseur de la couche a été mis en œuvre. Le principe de mesure de ces distributions de teneur en eau, basé sur une méthode de découpe destructive de l'échantillon, est décrit sur la figure 9 .

La couche est tout d'abord placée dans le dispositif expérimental de séchage (Fig. 1). Au bout d'un temps donné, l'expérience de séchage est stoppée et la cellule de mesure contenant la couche est immédiatement placée et maintenue dans le système de découpe grâce à deux vis de serrage (Fig. 9). Un piston dont on repère la position à l'aide d'une vis micrométrique permet de déplacer verticalement l'échantillon de sauce avec une grande précision et détermine ainsi l'épaisseur des tranches découpées par la lame de découpe. La cellule ayant été préalablement légèrement lubrifiée, cette opération n'induit que de faibles contraintes dans la couche. Chaque tranche découpée est ensuite pesée puis placée dans une étuve à $80^{\circ} \mathrm{C}$ pendant 24 heures pour 

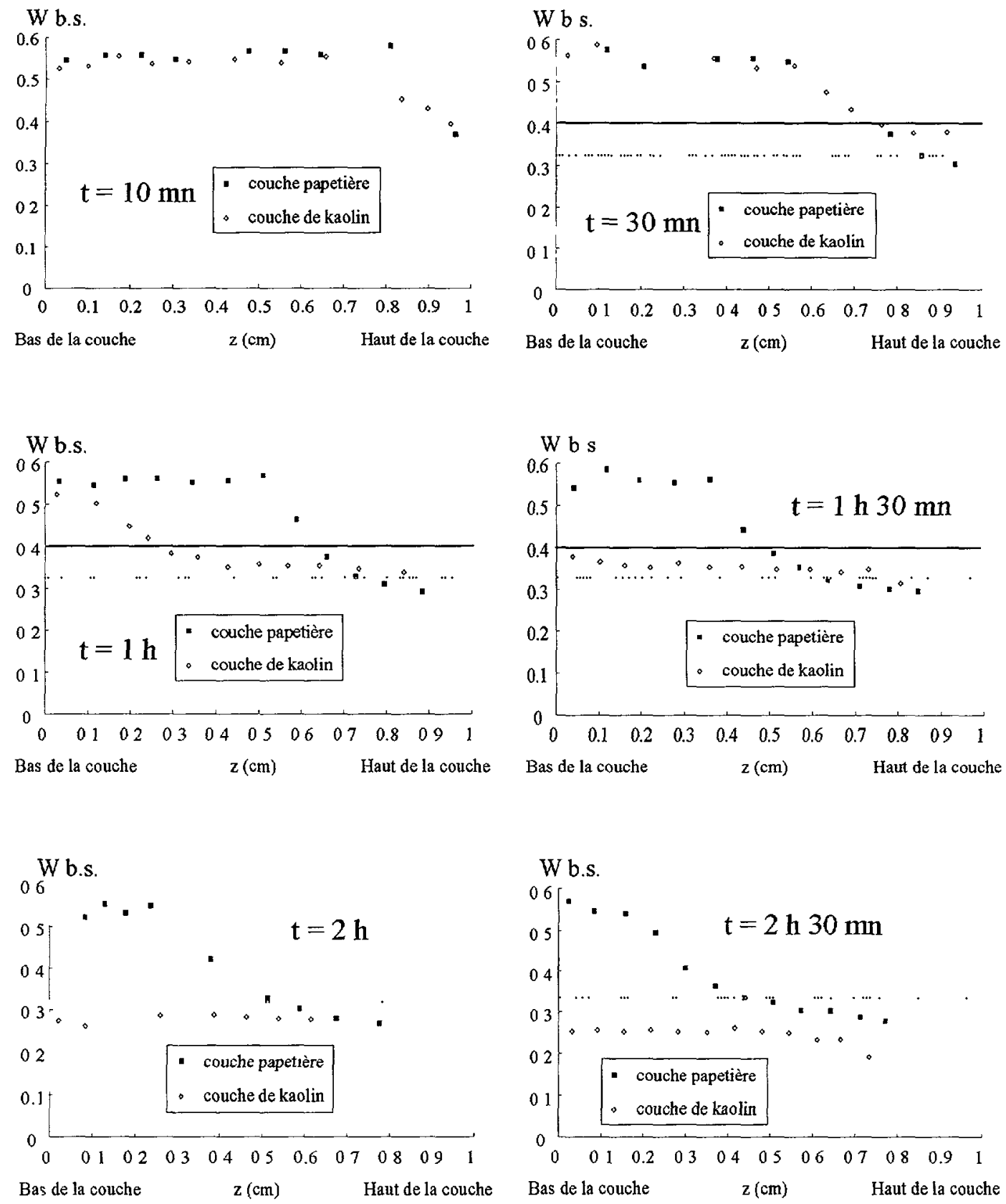

Fig. 10. - Profils de teneur en eau expérimentaux pour un séchage à $60^{\circ} \mathrm{C}$ sans simulation d'imbibition ; comparaison des profils obtenus pour une couche papetière et une conche de kaolin ; détermination graphique des valeurs des teneurs en eau au point de gel $W_{\mathrm{gp}}$ pour la couche papetière (pointillés) et $W_{\text {gk }}$ pour la couche de kaolin (trait continu).

[Moisture content profiles for a $60^{\circ} \mathrm{C}$ drying experiment without any impregnation simulation; kaolin coating profiles and paper coating profiles comparison; graphic determination of gel point moisture content values for a paper coating ( $W_{\mathrm{gp}}$, dashed line) and kaolin coating ( $W_{\mathrm{gk}}$, continuous line).] 

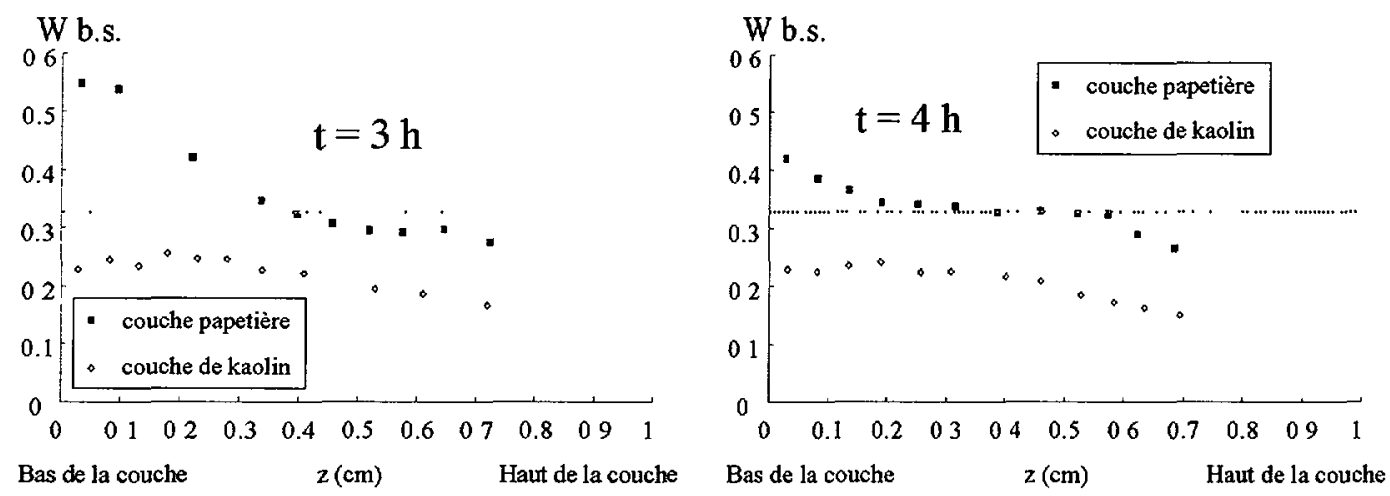

Fig. 10. - (Suzte.)

une déshydratation complète. La teneur en eau d'une lamelle est alors donnée par la relation

$$
W_{\imath}=\frac{m_{2}-m_{\mathrm{s} 2}}{m_{\mathrm{s} 2}}
$$

où $m_{\imath}$ et $m_{\mathrm{s} \imath}$ sont respectivement la masse totale et la masse sèche de la tranche $i$. L'opération de découpe et de pesée des tranches humides est suffisamment rapide pour que l'on considère qu'elle s'est effectuée à l'instant d'arrêt du séchage de la couche.

\subsubsection{Résultats expérimentaux}

2.3.2.1. Séchage sans simulation d'imbibition. - L'évolution des profils de teneur en eau au cours du séchage est présentée sur les figures 10 et 11 , respectivement pour des températures de séchage de $60^{\circ} \mathrm{C}$ et $80^{\circ} \mathrm{C}$ (manipulation sans simulation d'imbibition).

Afin d'appréhender l'influence de la présence des liants sur le transport interne de la phase liquide, deux séries de manipulations de séchage à $60^{\circ} \mathrm{C}$ ont été réalisées sur des couches papetières complètes et des couches composées uniquement d'eau et de kaolin. Dans les deux cas, la teneur en eau initiale a été fixée à 0,55 environ.

L'observation de ces figures amène trivialement à confirmer que la couche de kaolin sèche plus vite qu'une couche complète pour des conditions de séchage identiques. D'autre part, l'influence de la température de séchage sur l'évolution temporelle de ces profils est flagrante ; de plus, l'absence de simulation d'imbibition se traduit, comme souhaité, par une absence de séchage par le bas de la couche. Parallèlement au séchage et à la consolidation, le retrait de la couche prend effet dès le début de l'expérience. L'épaisseur de la couche diminue au cours du temps, pour finalement passer de $1 \mathrm{~cm}$ à environ $0,7 \mathrm{~cm}$ au bout de 4 heures de séchage.

Un point important concernant ces figures peut être souligné à ce niveau : pour les deux compositions de couche, on note l'apparition d'un front de séchage qui se propage à l'intérieur du matériau au fur et à mesure du séchage. La teneur en eau du bas de ce front est de l'ordre de 0,32 dans le cas d'une couche papetière (respectivement 0,4 dans le cas d'une couche de kaolin). Elle ne dépend pas de la température de séchage choisie dans la gamme de température que nous avons étudiée $\left(60^{\circ} \mathrm{C}-80^{\circ} \mathrm{C}\right)$. Elle est représentée sur les figures 10 et 11 , par un trait continu (couche de kaolin) et discontinu (couche papetière). Ce n'est que quand le front 

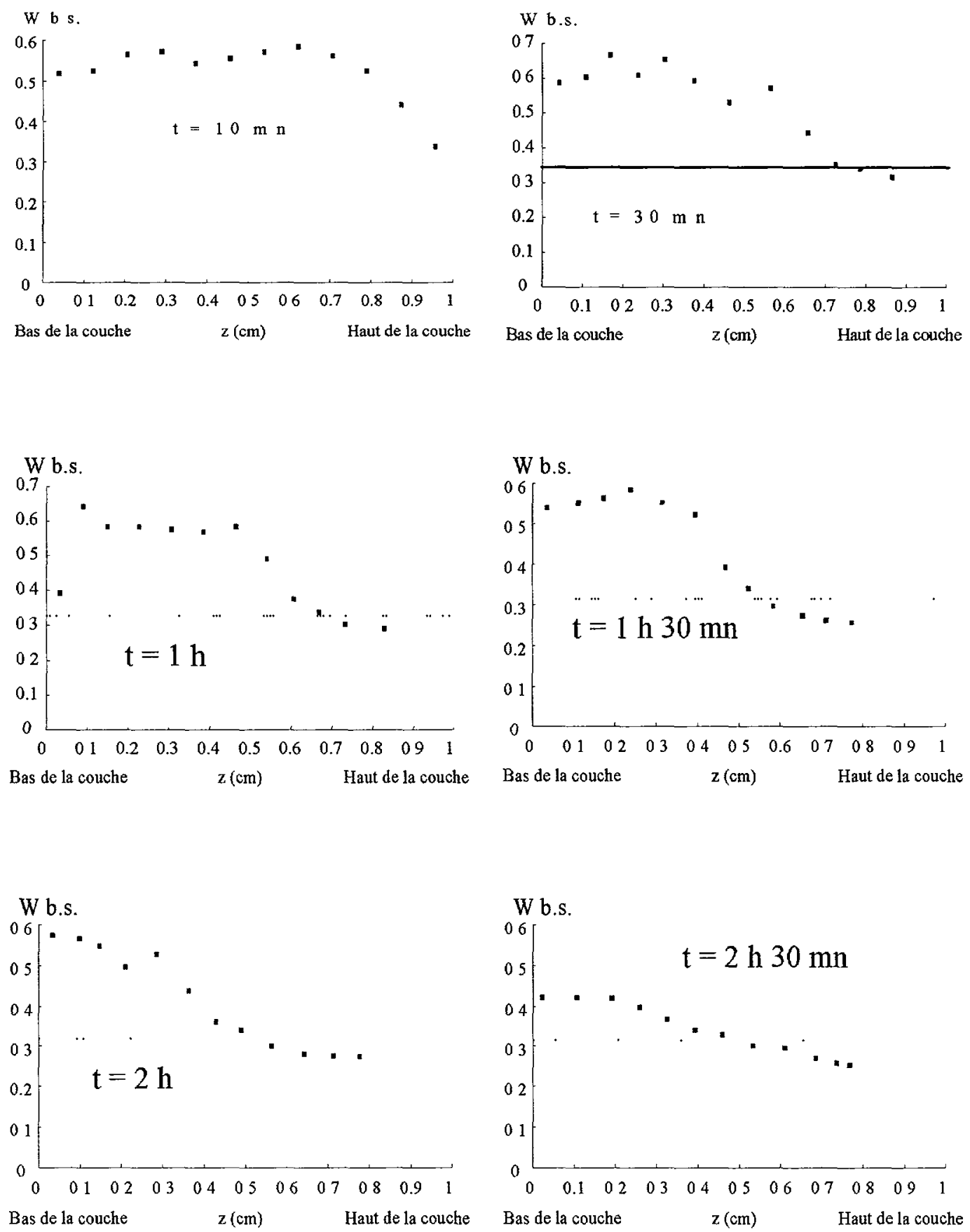

Fig. 11. - Profils de teneur en eau expérimentaux pour un séchage à $80^{\circ} \mathrm{C}$ sans simulation d'imbibition pour une couche papetière. Détermination de la valeur de la teneur en eau au point de gel $W_{g p}$ (pointillés).

[Moisture content profiles for a $80^{\circ} \mathrm{C}$ drying experiment without any impregnation simulation for a paper coating; graphic determination of gel point moisture content values ( $W_{\mathrm{gp}}$, dashed line).] 

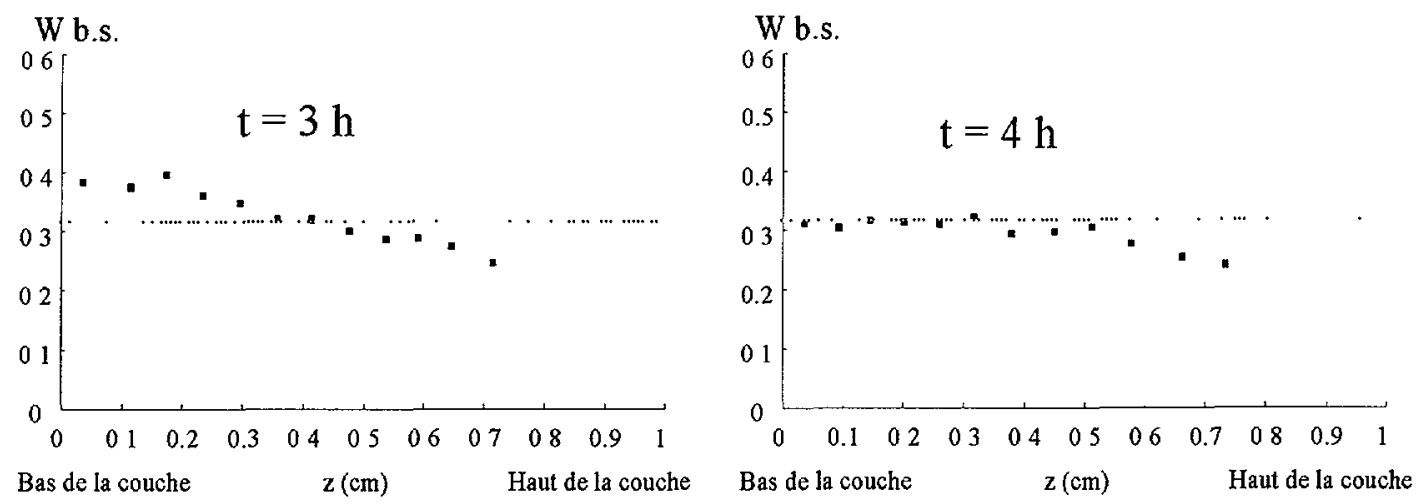

Fig. 11. - (Surte.)

de séchage a atteint le fond de la couche, c'est à dire quand la teneur en eau de la couche papetière est presque uniforme, que la couche recommence à perdre de l'eau en surface ; ainsi les transferts d'eau internes autour de cette teneur en eau de palier s'effectuent plus rapidement que pour des teneurs en eau supérieures (sauce liquide).

À ces teneurs en eau qui restent relativement élevées, les sauces utilisées sont encore diphasiques (liquide-solide). Par conséquent, ce front ne correspond pas à un front de séchage caractéristique de la pénétration de la phase gazeuse dans le matériau ; les teneurs en eau citées ci-dessus ne sont pas les teneurs en eau au point critique des sauces étudiées. Dans notre cas, ce front qui se propage lentement vers l'intérieur de la couche, matérialise la transition de la couche de l'état liquide vers l'état de gel. Ainsi, la teneur en eau au point de gel, caractéristique de la sauce utilisée peut donc ici être déduite par une simple lecture sur les profils de teneur en eau : $W_{\mathrm{gp}} \approx 0,32$ pour la couche papetière et $W_{\mathrm{gk}} \approx 0,4$ pour la couche de kaolin.

L'étude de la cinétique globale d'une couche papetière et d'une couche de kaolin (Fig. 6), nous a permis de montrer que le flux masse de séchage chute pour une teneur en eau moyenne égale à 0,30 et 0,23 respectivement pour la couche de kaolin et la couche papetière. Cette chute correspond à l'instant où le front de séchage pénètre dans le matériau, i.e. où la surface supérieure atteint le point critique (caractérisé par une teneur en eau locale $W_{c}$ ). En effet, lorsque le front de séchage est à l'intérieur du matériau, la vapeur issue de ce front a d'autant plus de difficulté à atteindre le dessus de la couche que l'épaisseur de milieu consolidé à traversée est grande. Ainsi, plus ce front rentre dans le matériau, plus le flux masse diminue.

Les profils de teneur en eau correspondant à la teneur en eau moyenne de 0,30 et 0,23 (couche de kaolin et couche papetière) sont tracés sur la figure 12. À l'aide de ceux-ci, la détermination du point critique s'effectue aisément en repérant la teneur en eau de surface de couche.

Ces profils font apparaître que la teneur en eau critique de la couche de kaolin $\left(W_{\mathrm{ck}} \approx 0,28\right)$ est supérieure à la teneur en eau critique caractéristique de la couche papetière $\left(W_{\mathrm{cp}} \approx 0,185\right.$ quelle que soit la température de séchage). Ces valeurs confortent l'analyse des cinétiques de séchage effectuée au paragraphe 2.2 ; en effet, comme nous le suggérions, $W_{\mathrm{ck}}>W_{\mathrm{cp}}$.

2.3.2.2. Séchage avec simulation d'imbibition. - L'évolution des profils de teneur en eau dans une couche papetière au cours d'une manipulation de séchage avec simulation d'imbibition, a été reportée sur les figures 13 et 14 , respectivement pour des séchages à $60^{\circ} \mathrm{C}$ et $80^{\circ} \mathrm{C}$. 


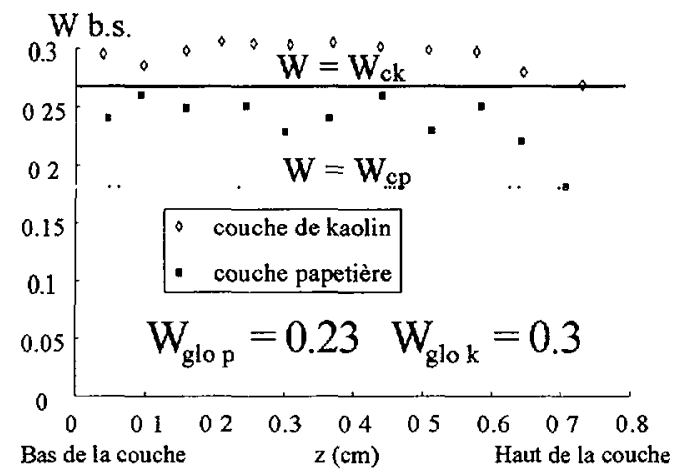

a)

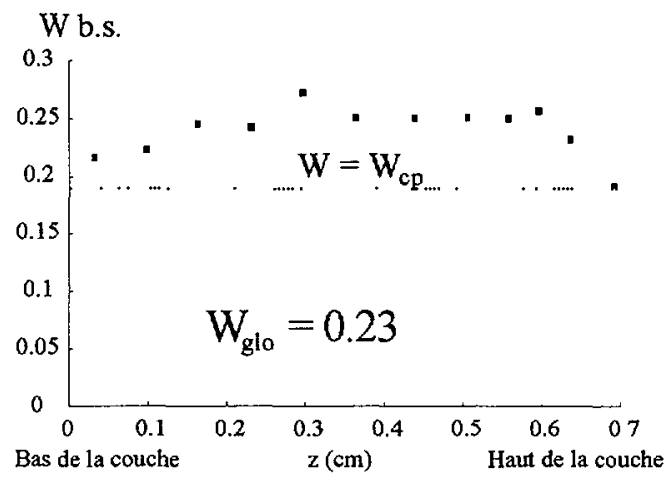

b)

Fig. 12. - Profils de teneurs en eau correspondant à : a) des teneurs en eau globales de 0,23 et 0,3 (couche papetière et couche de kaolin respectivement) pour un séchage à $60^{\circ} \mathrm{C}$; détermination des teneurs en eau critiques de la couche papetière $W_{\mathrm{cp}}$ (pointillés) et de la couche de kaolin $W_{\mathrm{ck}}$ (trait continu). b) une teneur en eau globale de 0,23 (couche papetière) pour un séchage à $80^{\circ} \mathrm{C}$; détermination de la teneur en eau critique de la couche papetière $W_{\mathrm{cp}}$ (pointillés).

[Moisture content profiles corresponding to a) 0.23 and 0.3 global moisture content of the paper and kaolin coating(s) respectively for a $60{ }^{\circ} \mathrm{C}$ drying experiment; graphic determination of critical point moisture content values for a paper coating ( $W_{\mathrm{cp}}$, dashed line) and a kaolin coating ( $W_{\mathrm{ck}}$, continuous line). b) 0.23 global moisture content (paper coating) for a $80^{\circ} \mathrm{C}$ drying experiment; graphic determination of critical point moisture content value ( $W_{\mathrm{cp}}$, dashed line).]

L'influence de la phase de vide sur le transport liquide est visible sur ces deux figures. En imposant brusquement une pression faible à la frontière inférieure de la couche, on crée un gradient de pression entre le haut et le bas de la couche qui favorise un transport liquide vers le fond de la cellule. Simultanément, on observe que la teneur en eau à l'interface couchemembrane chute brusquement pour atteindre une valeur stable. Cette valeur qui est maintenue durant toute la phase de vide, est de l'ordre de la teneur en eau au point de gel $\left(W_{\mathrm{g}} \approx 0,32\right)$. Chaque profil provenant d'une manipulation de séchage différente, il est clair que la répétitivité du phénomène observé montre bien qu'on n'observe pas là les conséquences d'erreurs expérimentales mais plutôt celles de processus physiques importants. Ceux-ci peuvent être interprétés de la façon suivante ; le fait d'imposer une pression de l'ordre de 0,1 bar à la frontière inférieure de la couche entraîne une augmentation brutale des transferts dans cette zone. L'intensité des échanges thermiques et massiques couplée à un transport liquide peu rapide dans la sauce provoque alors la chute brusque de la teneur en eau à la l'interface couche-membrane. Celle-ci atteint alors rapidement sa valeur de point de gel et ne change pas tant que presque toute la couche n'a pas atteint cette valeur.

Bien que ce ne soit pas notre propos ici, ce résultat est primordial pour la modélisation des transports de masse au sein de la couche papetière : il permet de prendre en compte les échanges massiques entre la couche et la chambre à vide par une condition à la limite de type teneur en eau imposée plutôt que flux imposé ; ainsi, l'obtention peu précise du flux masse d'imbibition expérimental n'est pas nécessaire pour simuler correctement le séchage d'une couche papetière [17]. 

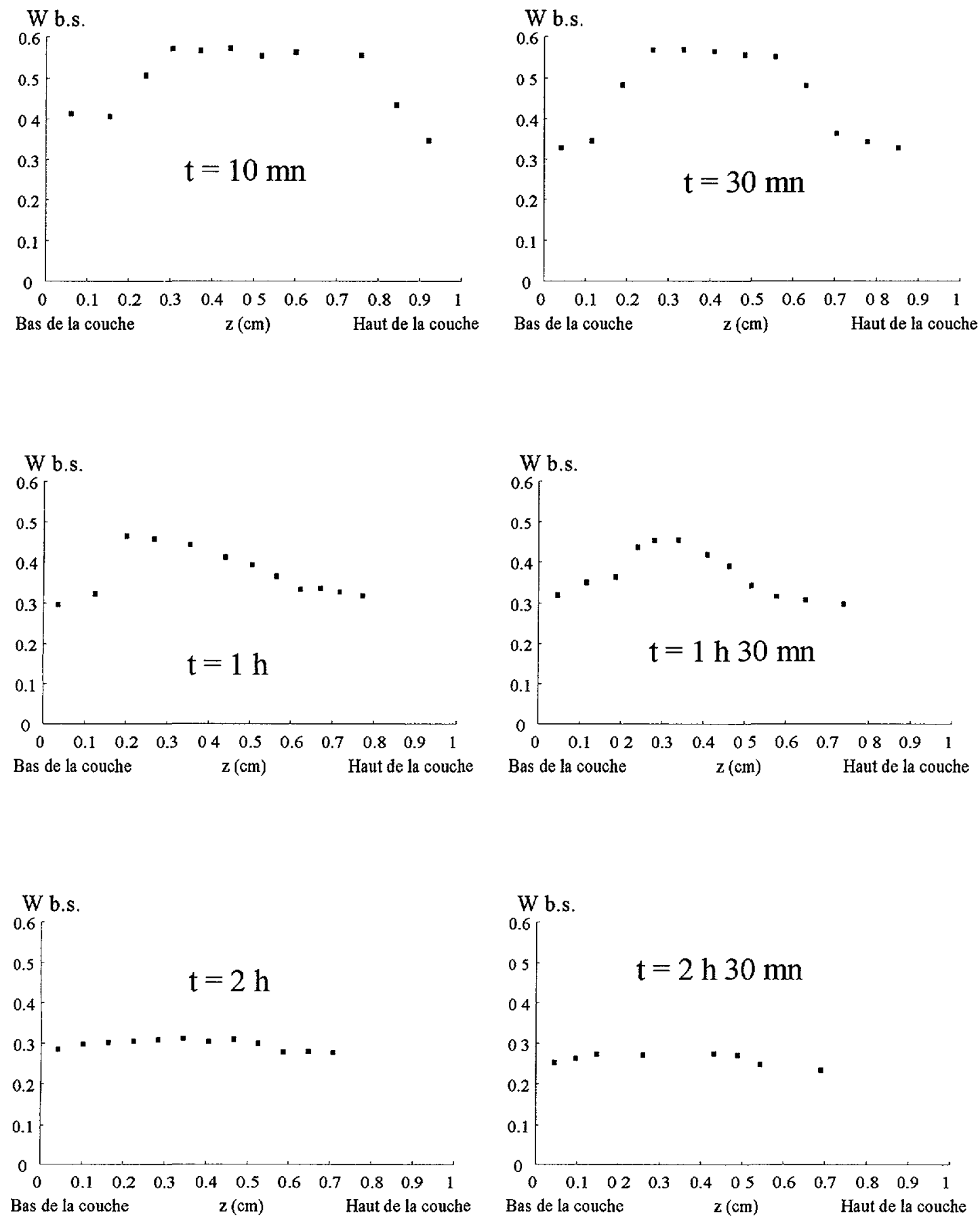

Fig. 13. - Profils de teneur en eau expérimentaux pour un séchage à $60^{\circ} \mathrm{C}$ d'une couche papetière avec simulation d'imbibition.

[Paper coating moisture content profiles with our impregnation simulation for a $60^{\circ} \mathrm{C}$ drying experiment. ] 

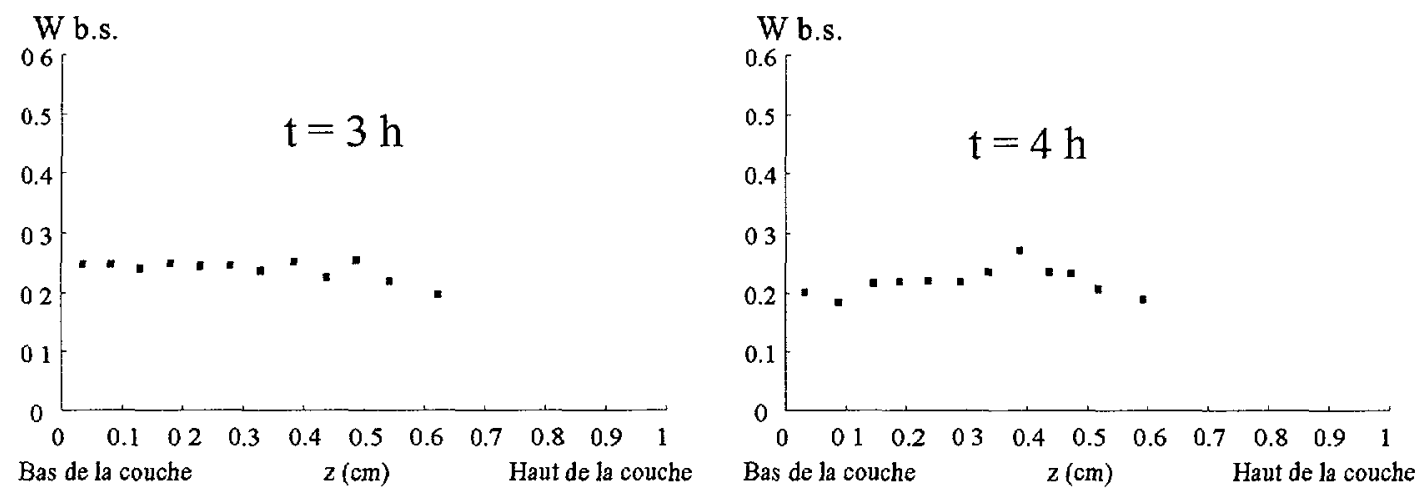

Fig. 13. - (Sunte.)
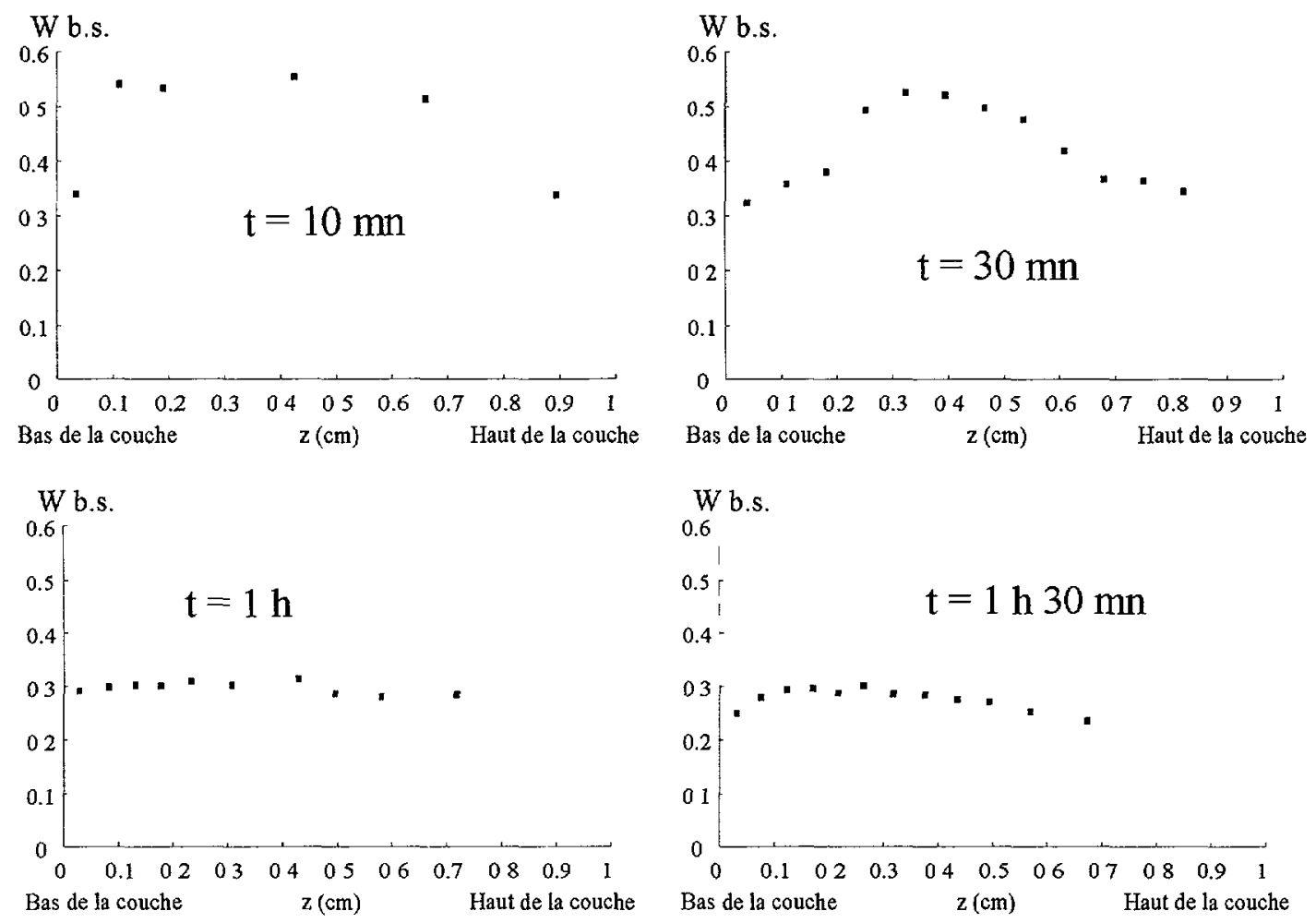

Fig. 14. - Profils de teneur en eau expérimentaux pour un séchage à $80^{\circ} \mathrm{C}$ d'une couche papetière avec simulation d'imbibition.

[Paper coating moisture content profiles with our impregnation simulation for an $80^{\circ} \mathrm{C}$ drying experiment.] 

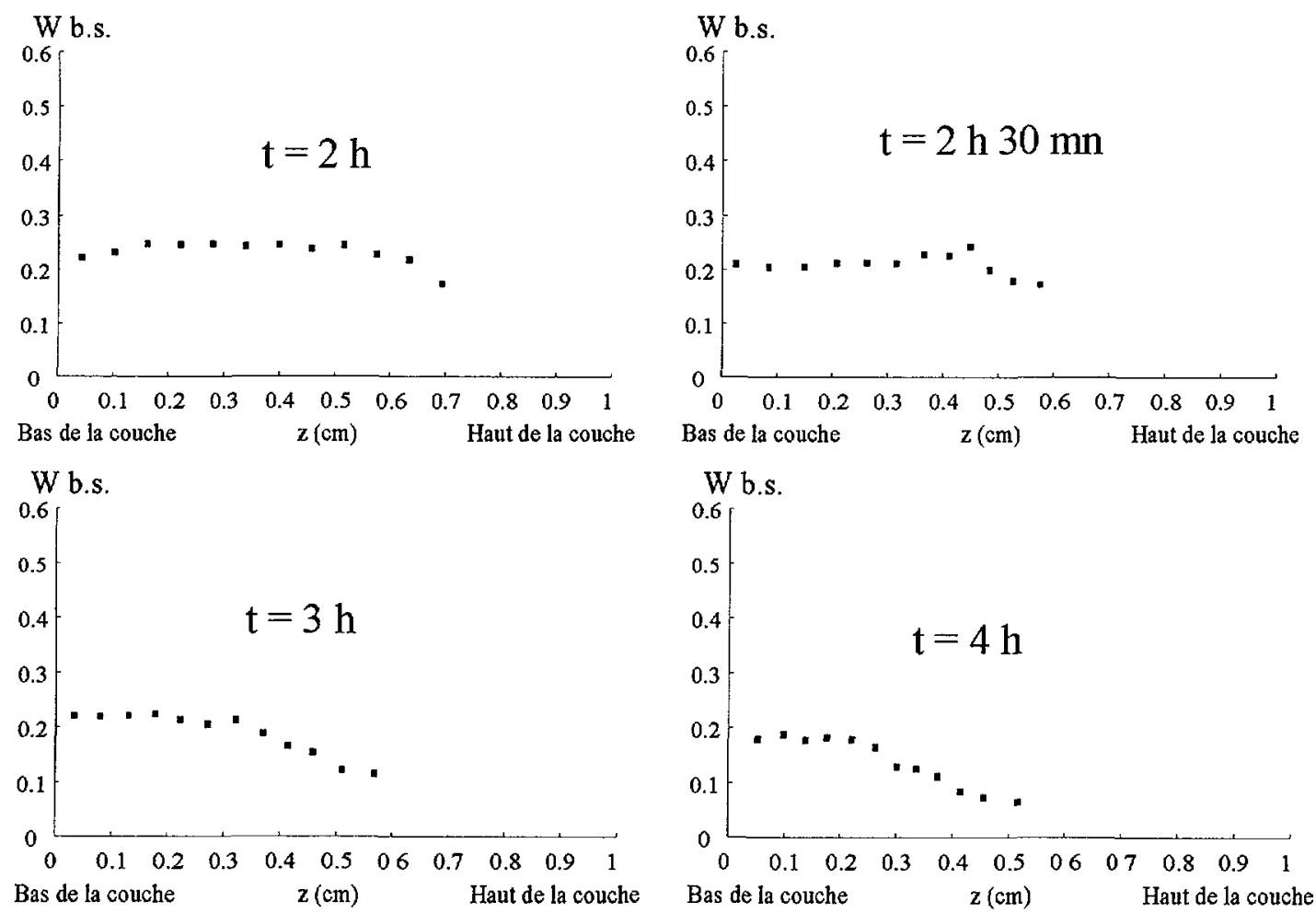

Fig. 14. - (Suite.)

\section{Analyse en terme de porosité de couche séchées}

Lors du séchage d'un matériau non consolidé comme une couche papetière, l'intensité du flux de séchage peut avoir une influence notable sur la structure intime finale du milieu consolidé. En particulier la porosité finale, le rayon moyen et la répartition de la taille des pores d'un échantillon de couche risquent d'être affectés par les conditions de séchage imposées. Or, ces caractéristiques géométriques de la couche séchée sont des paramètres déterminants dans la qualité et l'imprimabilité du papier : la couche doit être suffisamment poreuse pour permettre l'absorption rapide de l'encre lors de l'impression du papier.

Une série de manipulations expérimentales a donc été menée pour deux configurations de séchage et d'imbibition, sur des couches papetières de un centimètre d'épaisseur, ceci afin de déterminer les trois caractéristiques structurales de la couche consolidée citées ci-dessus.

Avant de passer aux résultats à proprement parler, une description du système expérimental utilisé pour les analyses porosimétriques est exposée.

3.1. PRinctpe de la mesure au porosimètre À Mercure. - L'appareil utilisé pour l'obtention de la porosité, du rayon moyen de pores, et de la distribution de la taille des pores est un porosimètre à mercure de marque CARLO ERBA, permettant de mesurer des rayons de pores compris entre $10^{-3}$ et $100 \mu \mathrm{m}$. Le principe de cette mesure est basé sur la relation 


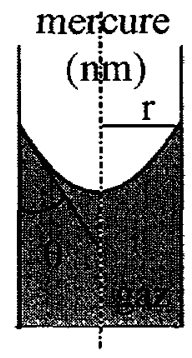

Fig. 15. - Représentation schématique d'un pore de rayon $r$ comprenant une phase liquide (mercure) et une phase gazeuse. Visualisation de l'angle de mouillage $\theta$.

[Schematic of a pore (radius $r$ ) full of liquid (mercury) and gas. Vizualisation of the wettability angle $\theta$.]

donnant la pression capillaire $P_{\mathrm{c}}$ dans un tube de section circulaire droite :

$$
P_{\mathrm{c}}=P_{\mathrm{nm}}-P_{\mathrm{m}}=\frac{2 \sigma \cos \theta}{r}
$$

où $r$ est le rayon du capillaire, $\sigma$ la tension interfaciale, $P_{\mathrm{nm}}$ et $P_{\mathrm{m}}$ les pressions dans la phase non mouillante et mouillante, $\theta$ l'angle de mouillage représenté sur la figure 15.

Une modélisation de la structure du milieu poreux testée par un ensemble de capillaires cylindriques parallèles de rayon $r$, permet d'établir une relation entre le volume de mercure ayant pénétré dans l'échantillon testé à une pression capillaire donnée et le rayon des pores pénétrés. Cette technique donne une idée de la répartition de la taille des pores sans toutefois rendre compte de la distribution réelle. En effet, le modèle simple de structure utilisé (réseau de capillaires parallèles) influence les résultats obtenus pour les répartitions de tailles de pores.

La procédure expérimentale est la suivante. De manière à accéder à une gamme de rayon de pores la plus large possible, deux types d'appareillages sont utilisés :

- Le premier donne accès aux macropores, pores dont le rayon est supérieur à $7,5 \mu \mathrm{m}$, en faisant varier la pression de $10^{-3}$ à 1 bar. Dans un premier temps, l'échantillon de couche sèche est placé dans un dilatomètre à mercure dans lequel le vide est réalisé ; ce dilatomètre est constitué d'un réservoir prolongé dans sa partie haute par un capillaire calibré. Dans un deuxième temps, la cellule est remplie de mercure, toujours sous vide. Une fois cette étape franchie, la série de mesure peut commencer : à l'aide d'une vanne permettant l'arrivée d'air, la pression est incrémentée par paliers dans le dilatomètre et le mercure pénètre dans l'échantillon ; ainsi le niveau de mercure dans le capillaire calibré baisse. La pression appliquée et le niveau de mercure dans le capillaire complétant la cellule sont repérés manuellement.

- Lorsque la pression atmosphérique est atteinte, la cellule est placée dans un autoclave. Progressivement le système est entouré d'huile haute pression faisant office de fluide intermédiaire pour l'application de la pression de 1 à 4000 bars. Lors de cette montée en pression, le niveau de mercure dans le capillaire est repéré par une mesure électrique capacitive que nous ne décrivons par ici. Cette partie est totalement automatisée. Elle permet l'accès aux pores dont le rayon est compris entre $10^{-3}$ et $7,5 \mu \mathrm{m}$. 
- Un logiciel (MILESTON), basé sur un modèle de capillaires cylindriques est associé au porosimètre. Il permet, à partir de la mesure de la quantité de mercure qui pénètre dans le matériau à chaque palier de pression dans ce mercure, de remonter à la porosité finale, au rayon moyen de pores et la répartition de la taille des pores dans l'échantillon testé.

3.2. ÉTUDE DE LA POROSITÉ MOYENNE DES COUCHES. - À l'aide du dispositif expérimental décrit précédemment, une série d'expériences de porosimétrie sur des couches séchées sous différentes conditions de séchage et d'imbibition a été menée. Chaque expérience consiste en une analyse porosimétrique de cinq tranches horizontales découpées dans la couche considérée.

Il est possible de comparer la valeur de la porosité moyenne d'une tranche déterminée à l'aide du porosimètre à mercure, à celle de la porosité théorique obtenue à l'aide de la connaissance de la concentration volumique $\phi_{c}$ en matière solide au point critique. Cette concentration volumique en matière solide ne varie plus au delà du point critique : en effet, par définition, au delà du point critique, la sauce est consolidée et elle ne se contracte plus (retrait nul). Ainsi, en gardant en mémoire qu'au point critique le milieu est encore diphasique, $\phi_{\mathbf{c}}$ s'exprime comme suit:

$$
\phi_{\mathrm{c}}=\frac{\rho_{\ell}}{\rho_{\mathrm{s}} W_{\mathrm{c}}+\rho_{\ell}}
$$

où $\rho_{\ell}$ et $\rho_{\mathrm{s}}$ sont respectivement la masse volumique de l'eau et la masse volumique moyenne de la phase solide. Malgré les migrations d'amidon observées, cette dernière est supposée uniforme dans une couche papetière car la proportion massique en amidon dans la sauce de couchage est minime (voir paragraphe 4).

En fin de séchage, la couche est composée de matière solide et de phase gazeuse. La fraction volumique de gaz n'est autre que la porosité de la couche $(\varepsilon)$ et ainsi,

$$
\varepsilon=1-\phi_{\mathrm{c}}
$$

Les résultats concernant les profils de porosité moyenne mesurés par porosimétrie au mercure dans des couches papetières soumises à diverses conditions de séchage sont reportés sur la figure 16 et comparés à la valeur de la porosité déduite de la connaissance du point critique. Le même type de résultats est aussi tracé pour une couche de kaolin séchée à $70^{\circ} \mathrm{C}$.

Ces résultats montrent bien l'effet de fermeture des liants sur la porosité finale de la couche. Ces liants, par leur action sur la charge d'une couche papetière, accentuent la contraction de celle-ci et en diminuent la porosité moyenne finale.

De plus, que ce soit pour une couche papetière ou une couche de kaolin, la porosité moyenne mesurée et celle calculée à l'aide de l'équation (3.3) concordent. Ce résultat permet notamment de valider les différentes définitions du point critique suggérées dans la littérature et utilisées pour la détermination graphique de la valeur de la concentration critique : le point critique est atteint en surface de couche au moment où la couche papetière rentre dans une seconde période de ralentissement (deuxième chute du flux masse). À partir de cet instant, le front de séchage pénètre dans la couche (apparition d'une phase gazeuse) ; la partie de la couche située entre ce front et le sommet de la couche est un solide indéformable. 


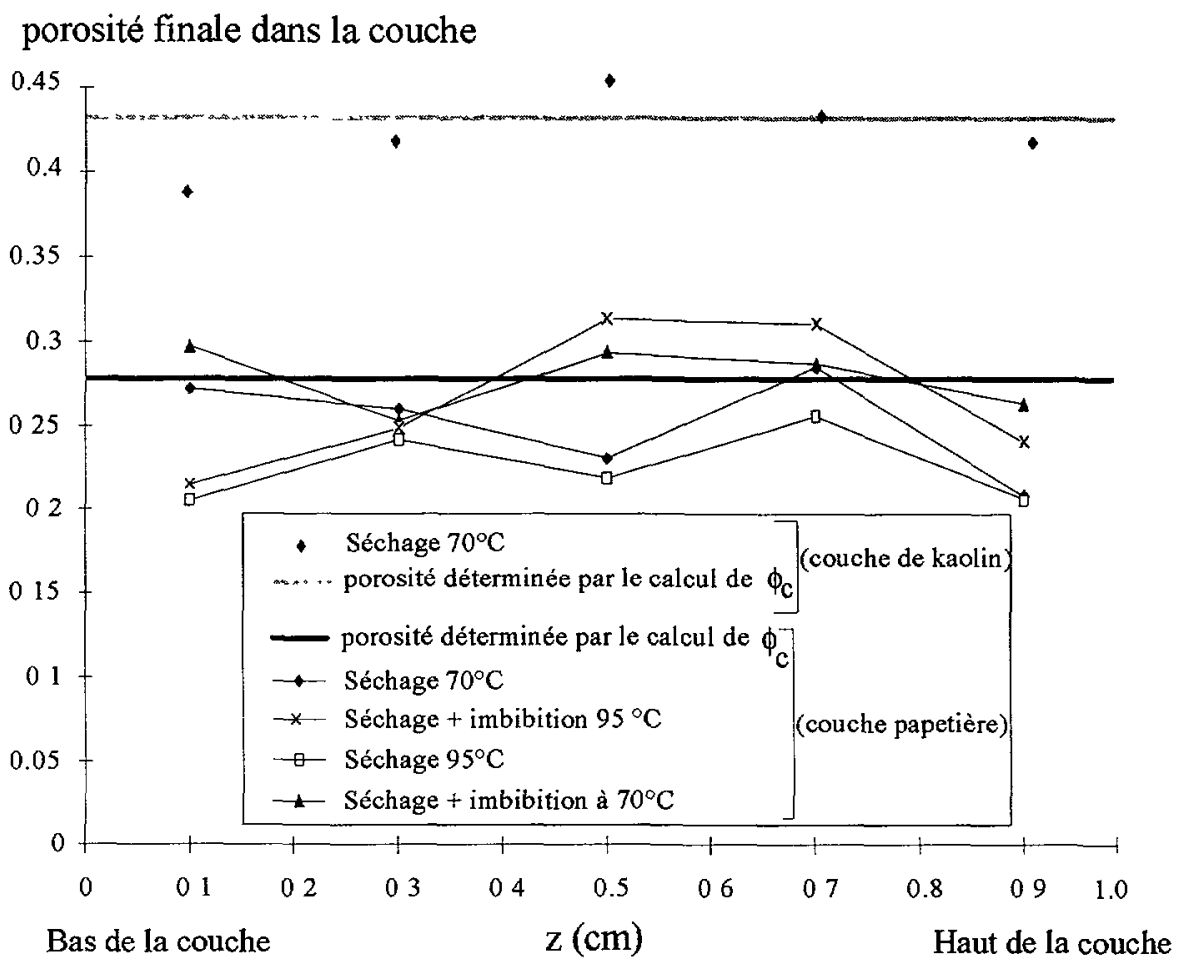

Fig. 16. - Profils expérimentaux de porosité au sein de la couche; comparaison avec la valeur de la porosité déduite de la valeur de la concentration volumique en constituants solides au point critique.

[Porosity profiles in a dried coating; comparison with the porosity calculated from the critical volume concentration of solid components. ]

3.3. Étude des PROFILS DE RÉPARTITION DE TAILlES DE PORES DANS LA COUCHE. Les répartitions spatiales de la taille des pores sont représentées en annexe pour une couche papetière séchée avec les conditions de séchage suivantes :

$\star$ Figure 20 : Séchage à $70^{\circ} \mathrm{C}$ sans simulation d'imbibition.

* Figure 21 : Séchage à $95^{\circ} \mathrm{C}$ sans simulation d'imbibition.

* Figure 22 : Séchage à $70^{\circ} \mathrm{C}$ avec simulation d'imbibition.

* Figure 23 : Séchage à $95^{\circ} \mathrm{C}$ avec simulation d'imbibition.

Les tranches sont numérotées de 1 (bas de la couche) à 5 (haut de la couche). Avant d'étudier plus en détail ces deux séries de figures, il convient de préciser la démarche adoptée pour obtenir ces courbes : les résultats bruts qui nous sont donnés par le logiciel MILESTONE sont traduits en terme de volume cumulé entré dans l'échantillon $(V)$ à une pression donnée en fonction du rayon du pore pénétré à cette pression. En dérivant ce volume par rapport au rayon (terme $\mathrm{d} V / \mathrm{d} r$ tracé en ordonnée sur les figures), on obtient la courbe de répartition spatiale de la taille des pores. 
À la lecture de ces résultats, deux points essentiels peuvent être dégagés :

1- Le rayon moyen de pores des échantillons est indépendant des conditions de séchage et d'imbibition. Celui-ci a pour valeur $0,035 \pm 0,004 \mu \mathrm{m}$.

2- On distingue approximativement deux types de répartitions différentes, associées dans chaque cas à des conditions de séchage et d'imbibition bien précises :

- Lorsque le flux masse local est relativement faible en début de séchage (avant la gélification locale de la sauce), la distribution des rayons de pore est en forme de pic ; ceci traduit une homogénéité de la porosité de la tranche considérée. Une analyse des cinétiques et des évolutions temporelles des profils de teneurs en eau obtenues pour les différentes conditions de séchage testées nous a permis de déterminer les tranches qui ont subit un flux masse faible avant leur passage à l'état de gel : ce sont toutes les tranches pour un séchage pur à $70^{\circ} \mathrm{C}$, les tranches 2 à 4 pour un séchage pur à $95^{\circ} \mathrm{C}$, les tranches 2 à 5 pour un séchage avec imbibition à $70^{\circ} \mathrm{C}$, et les tranches 3 et 4 pour un séchage avec imbibition à $95^{\circ} \mathrm{C}$. En résumé, ce sont les tranches qui n'ont, ni subit un fort flux de séchage (tranche 5 quand le séchage s'effectue à $95^{\circ} \mathrm{C}$ ), ni subit un flux d'imbibition (plus ou moins accentué selon la température de séchage du fait de la viscosité de la sauce), ni subit un drainage par le bas du fait de la dilatation thermique de la couche (tranche 1 pour un séchage à $95^{\circ} \mathrm{C}$ ).

- Lorsque le flux masse local est plus fort en début de séchage, la répartition spatiale de la taille des pores est étalée. Cet "étalement" correspond à une forte hétérogénéité de porosité de la tranche considérée.

Ces résultats montrent que le degré d'hétérogénéité de la porosité de surface de la couche est fortement lié à l'intensité du flux masse d'évaporation ou d'imbibition. Ceci est en accord avec les travaux expérimentaux de Takase et al. [18] qui proposent un modèle qualitatif pour la consolidation des couches lors du séchage. Ces auteurs montrent que lors d'un séchage doux, les pigments présents dans la couche s'orientent horizontalement pour former au fur et à mesure du séchage, une structure de mieux en mieux organisée et une couche finale à porosité homogène. Au contraire, lorsque le flux de séchage est important, les liants forment rapidement un film de telle sorte que la structure formée par les pigments se solidifie avant que ceux-ci n'atteignent leur orientation horizontale. La structure solide est alors fortement désorganisée ce qui entraîne de nombreuses hétérogénéités dans la porosité de la couche (voir Fig. 17). Dans la couche papetière utilisée, les kaolins qui représentent $25 \%$ en masse de la sauce humide ont des formes de plaquettes et ont donc des difficultés à se ranger uniformément lors de forts flux masse locaux. Il n'est donc pas surprenant d'observer un étalement de l'histogramme de répartition de tailles de pores pour de tels flux masse locaux initiaux.

Dans chacun des essais réalisés sur une couche papetière, l'ordre de grandeur du rayon maximum des pores de l'échantillon de couche papetière est toujours inférieur au micromètre et de plus, la plupart des pores ont un rayon compris entre 0,01 et $0,1 \mu \mathrm{m}$. D'autres essais de porosimétrie ont été réalisés sur des couches de kaolin séchées à $70^{\circ} \mathrm{C}$ sans simulation d'imbibition (Fig. 18) : le rayon moyen équivalent des pores de la couche de kaolin est alors de l'ordre de $1,75 \mu \mathrm{m}$ et $90 \%$ des pores ont un rayon compris entre 0,1 et $2 \mu \mathrm{m}$; l'effet des liants sur la fermeture de la couche papetière est donc important. 


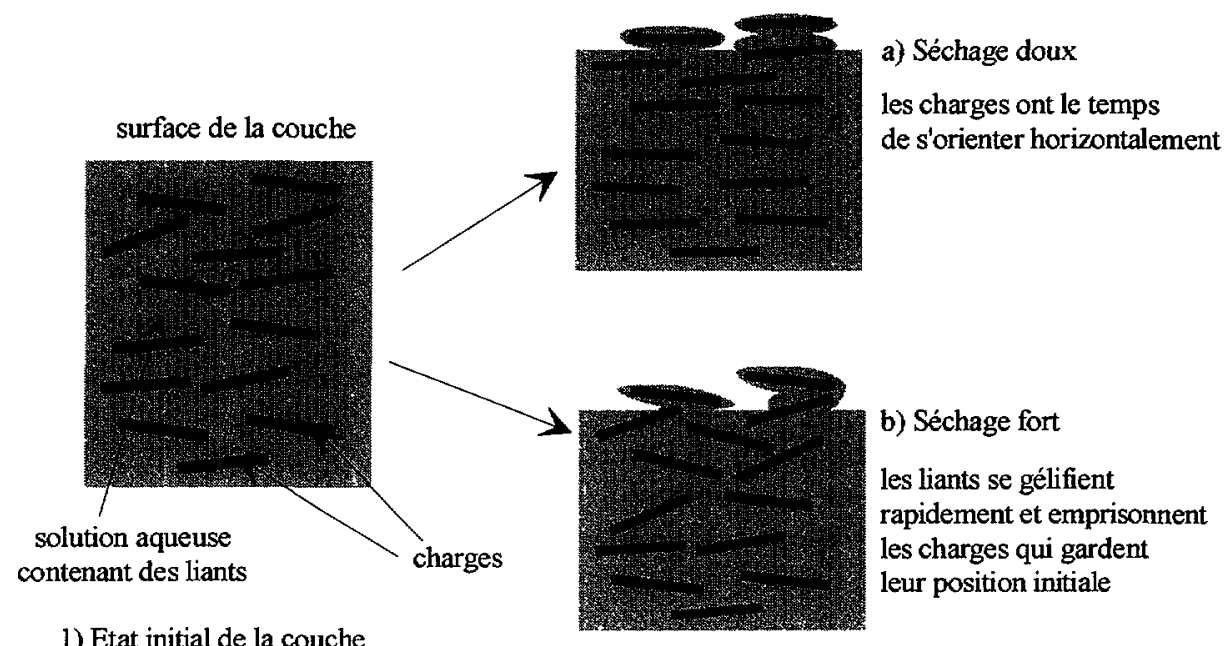

2) Etat de la couche après le point critique

Fig. 17. - Schématisation de l'évolution de la structure de la couche au cours du séchage (d'après Takase et al. [16]).

[Schématization of the coating structure evolution during drying (from Takase et al. [16]).]

Les travaux de Moyne [13] mettent l'accent sur une classification des milieux poreux suivant la répartition de la taille des pores qui le constituent. L'auteur met ainsi en évidence un caractère hygroscopique ou non du matériau, caractère qu'il associe à la taille de pores du milieu. Dans notre cas, la couche papetière n'est constituée que de pores de rayon inférieur au micromètre et le matériau est donc hygroscopique. Dans le cas d'une couche simple de kaolin, tous les pores ont un rayon compris entre 0,09 et $2 \mu \mathrm{m}$, et on peut alors considérer que ce milieu est non hygroscopique. Ce caractère non hygroscopique de la couche de kaolin a déjà été vérifié sur la cinétique de séchage (paragraphe 2.2.1).

On aboutit donc à la conclusion que la présence des liants, confère à la couche un caractère hygroscopique que n'a pas une simple couche de kaolin. En particulier les composants hydrophiles des liants (liants naturels tels que l'amidon) peuvent retenir l'eau sous forme adsorbée. Cette eau fortement liée à la structure intime de la macromolécule d'amidon devra être extraite de la matrice solide pour ensuite être évaporée.

\section{Analyse de la migrations de l'amidon dans une couche séchée}

$\mathrm{Au}$ cours du séchage, le flux d'évaporation et le flux d'imbibition induisent une migration du liquide respectivement vers le haut et vers le bas de la couche. Le transport des liants à travers la couche et en particulier celui de l'amidon, est intimement lié au mouvement de la phase liquide. Cette migration de liants joue un rôle non négligeable sur la qualité finale et l'imprimabilité du papier couché ; ainsi, il nous est apparu important de nous intéresser au transport d'un liant fortement lié à l'eau car hydrophile, l'amidon.

Le principe de la méthode utilisée pour le dosage de l'amidon dans un échantillon de couche est basé sur l'extraction de l'amidon par une solution d'acide chlorhydrique, suivi de la détermination de la concentration d'amidon par colorimétrie à l'iode et mesure au spectrophotomètre 


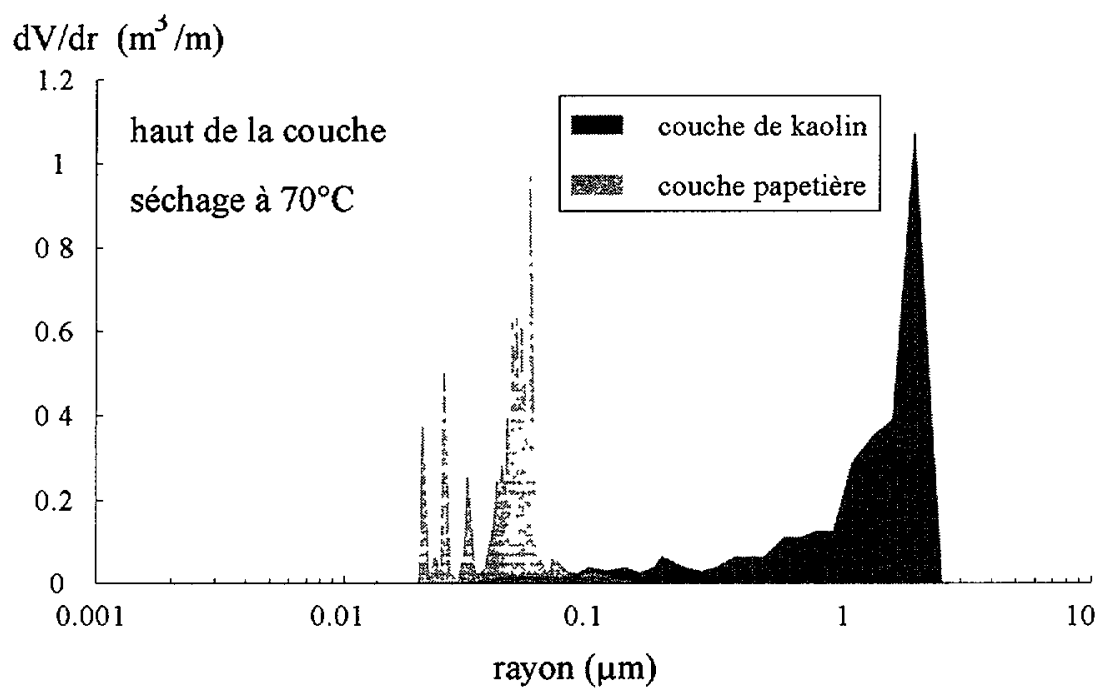

Fig. 18. - Comparaison des répartitions spatiales des rayons des pores d'une couche de kaolin et d'une couche papetière séchés à $70^{\circ} \mathrm{C}$ sans simulation d'imbibition.

[Pore size distribution comparison between a kaolin and a paper coating for a $70^{\circ} \mathrm{C}$ drying experiment without any impregnation simulation.]

UV-visible. Dans ce paragraphe, nous ne donnerons pas de description détaillée des dispositifs expérimentaux classiques employés.

Pour chaque tranche testée, quatre dosages sont effectués et la concentration retenue est la moyenne des quatre concentrations obtenues pour les différents dosages.

Les résultats de ces expériences de dosage sont reportés sur la figure 19, pour six configurations de séchage et d'imbibition différentes. L'abscisse de ces figures est adimensionnalisée par rapport à $e_{\text {tot }}$ (épaisseur de la couche séchée) et l'ordonnée par rapport à $W_{\mathrm{a}_{\text {moy }}}$ (concentration massique moyenne en amidon dans la couche considérée).

Quelle que soit la température de séchage, une forte proportion d'amidon est présente à la surface supérieure de la couche en fin de séchage. Ainsi, il apparaît clairement qu'une partie de l'amidon est transportée par la phase liquide au cours du séchage. Ce phénomène se produit même pour des conditions de séchage très douces. Pour des conditions plus sévères $\left(60^{\circ} \mathrm{C}\right.$ et $80^{\circ} \mathrm{C}$ ), la concentration d'amidon en surface de la couche est croissante avec la température de séchage.

L'effet du vide imposé au bas de la couche est aussi visible sur les courbes de la figure 19 : la proportion massique d'amidon en fin de séchage est en effet supérieure à la moyenne à la frontière inférieure de la couche, dans le cas où l'imbibition a été simulée. Le transport liquide créé par le flux d'imbibition dirigé vers le bas, entraîne donc bien une partie de l'amidon vers la frontière inférieure de la couche. Toutefois, du fait que la phase de vide ne dure qu'une heure, l'influence du flux d'imbibition sur les répartitions d'amidon en fin d'expérience est moins visible que celle du flux de séchage. 

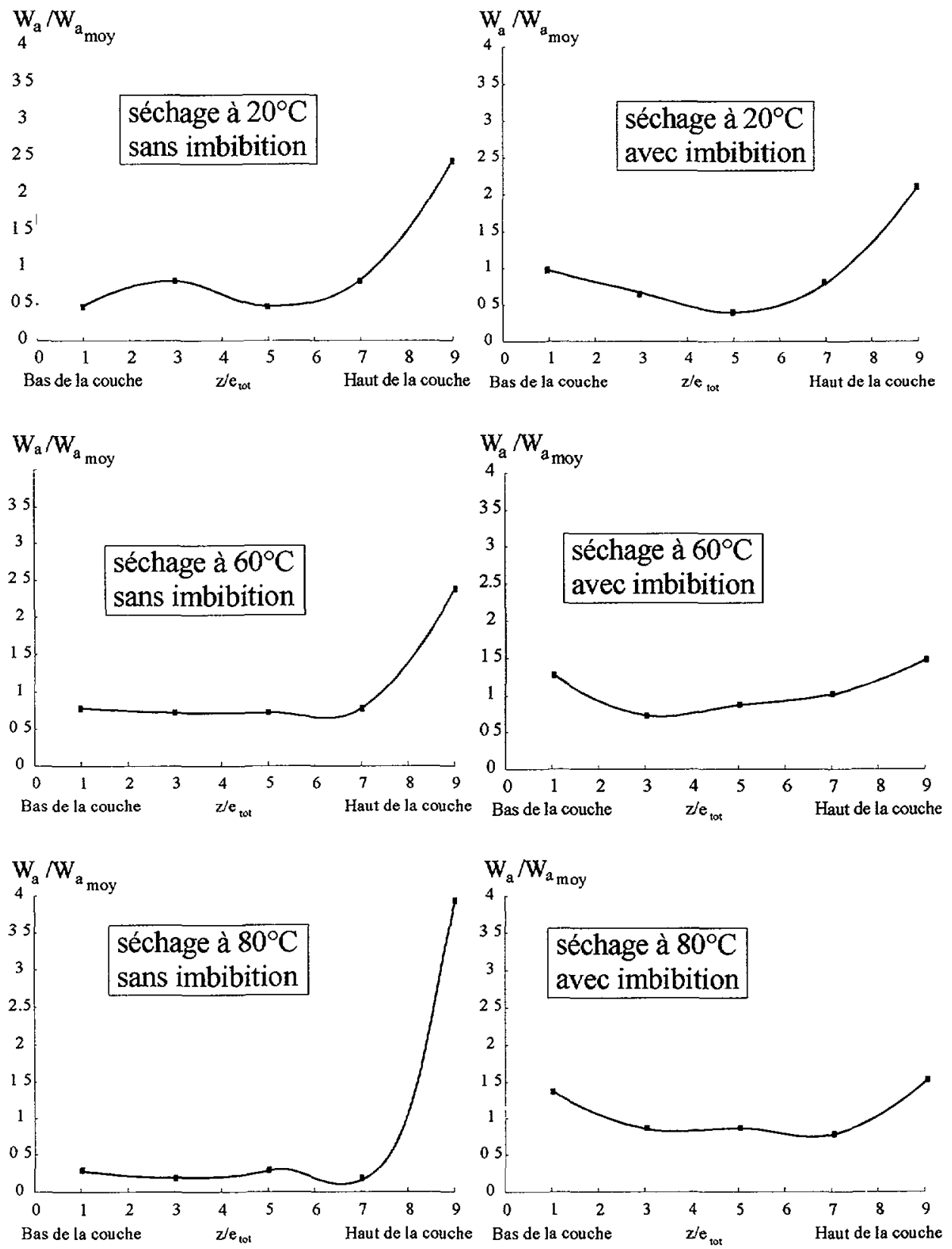

Fig. 19. - Profils de concentration d'amidon en fin de séchage dans une couche papetière ; influence des conditions de séchage et d'imbibition sur le transport de l'amidon au sein de la couche.

[Starch concentration profiles in a dried paper coating; the drying and impregnation conditions influence on the starch transport in the paper coating.] 


\section{Conclusion}

Au terme de cette étude expérimentale consacrée au séchage d'une couche papetière d'épaisseur initiale $1 \mathrm{~cm}$, quelques éléments importants peuvent être dégagés.

1) l'analyse des cinétiques expérimentales de séchage a permis de mettre en évidence l'existence de trois phases de séchage.

- Une première phase de ralentissement pendant laquelle le flux masse diminue sensiblement. Une analyse comparative des cinétiques de séchage d'une couche simple de kaolin et d'une couche papetière complète, montre que la chute du flux masse est due à la gélification des liants dans la couche.

- Une deuxième phase qui correspond à une phase quasi-isenthalpe de séchage et durant laquelle le flux masse reste constant.

- Une troisième phase à partir de laquelle le flux masse chute à nouveau (deuxième phase de ralentissement) ; celle-ci débute au moment où la surface atteint le point critique à partir duquel la structure intime de la couche passe de l'état de gel à l'état de solide indéformable. À cet instant, le front de séchage pénètre dans le matériau et la porosité finale de la couche commence à se construire.

2) L'étude sur les cinétiques d'imbibition a permis de valider le montage expérimental réalisé pour simuler l'imbibition.

3) La détermination des profils expérimentaux de teneur en eau a permis la mesure des teneurs en eau caractéristiques des changements d'état de la sauce (point de gel et point critique).

4) Une étude porosimétrique des couches séchées sous différentes conditions de séchage et d'imbibition a amené à déterminer l'influence du flux masse sur l'homogénéité de la porosité de la couche : plus le flux masse est important en début de séchage (forte température de séchage ou imbibition), plus la porosité des surfaces de la couche est hétérogène.

5) Enfin, l'analyse des profils de concentration massique d'amidon en fin de séchage a montré que l'amidon est transporté par la phase liquide vers les surfaces de la couche, et ce d'autant plus nettement que le flux masse local imposé par les conditions de séchage et de simulation de l'imbibition est élevé durant le séchage (quand la sauce n'est localement pas encore gélifiée).

\section{Annexe}

Détermination de la répartition de la taille des pores dans un enduit de couchage papetier sous différentes conditions de séchage (Figs. 20 à 23, pages suivantes). 

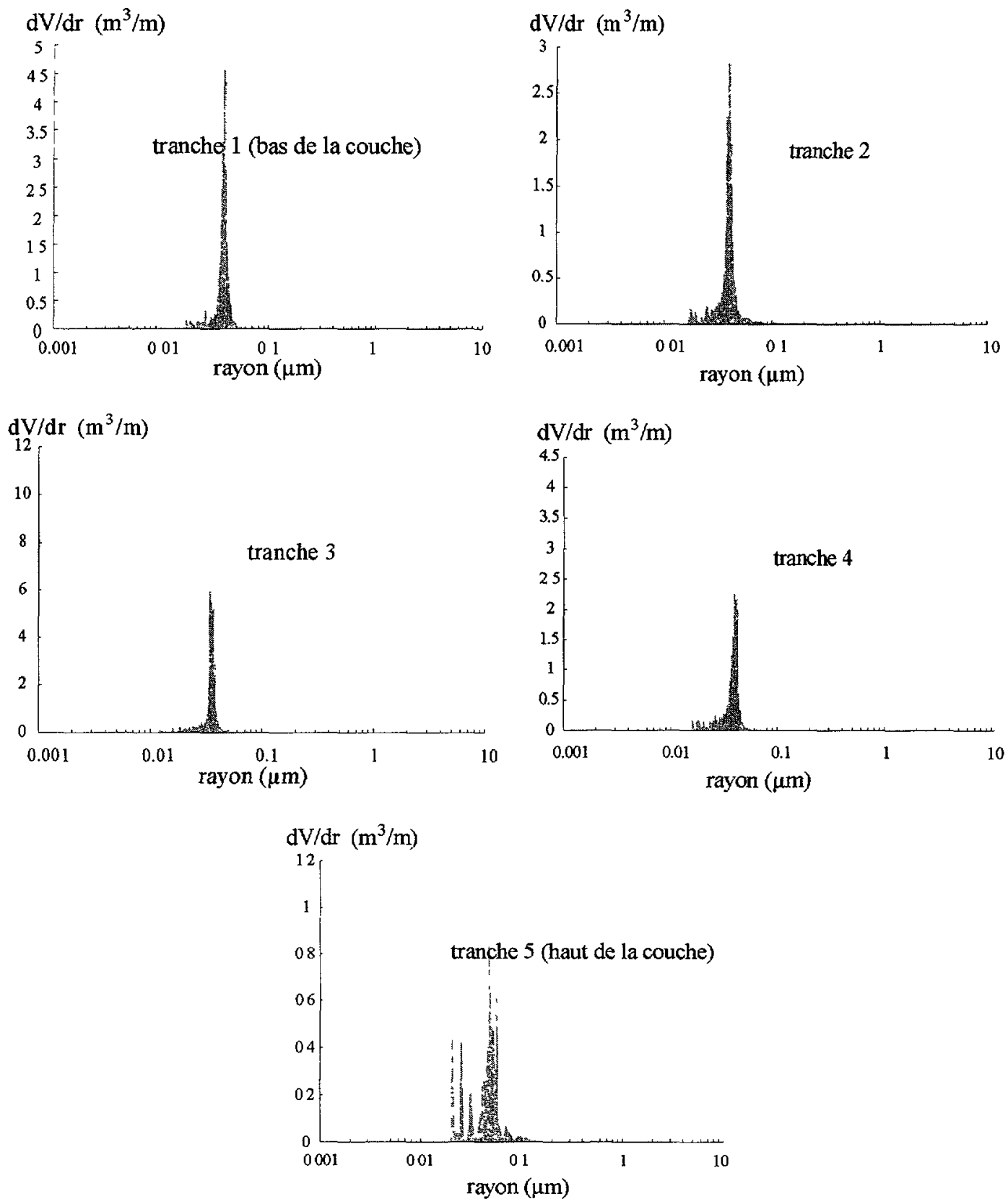

Fig. 20. - Répartition de la taille des pores dans une couche séchée à $70^{\circ} \mathrm{C}$ sans simulation d'imbibition.

[Pore size distribution in a paper coating; a $70{ }^{\circ} \mathrm{C}$ drying experiment without any impregnation simulation. ] 

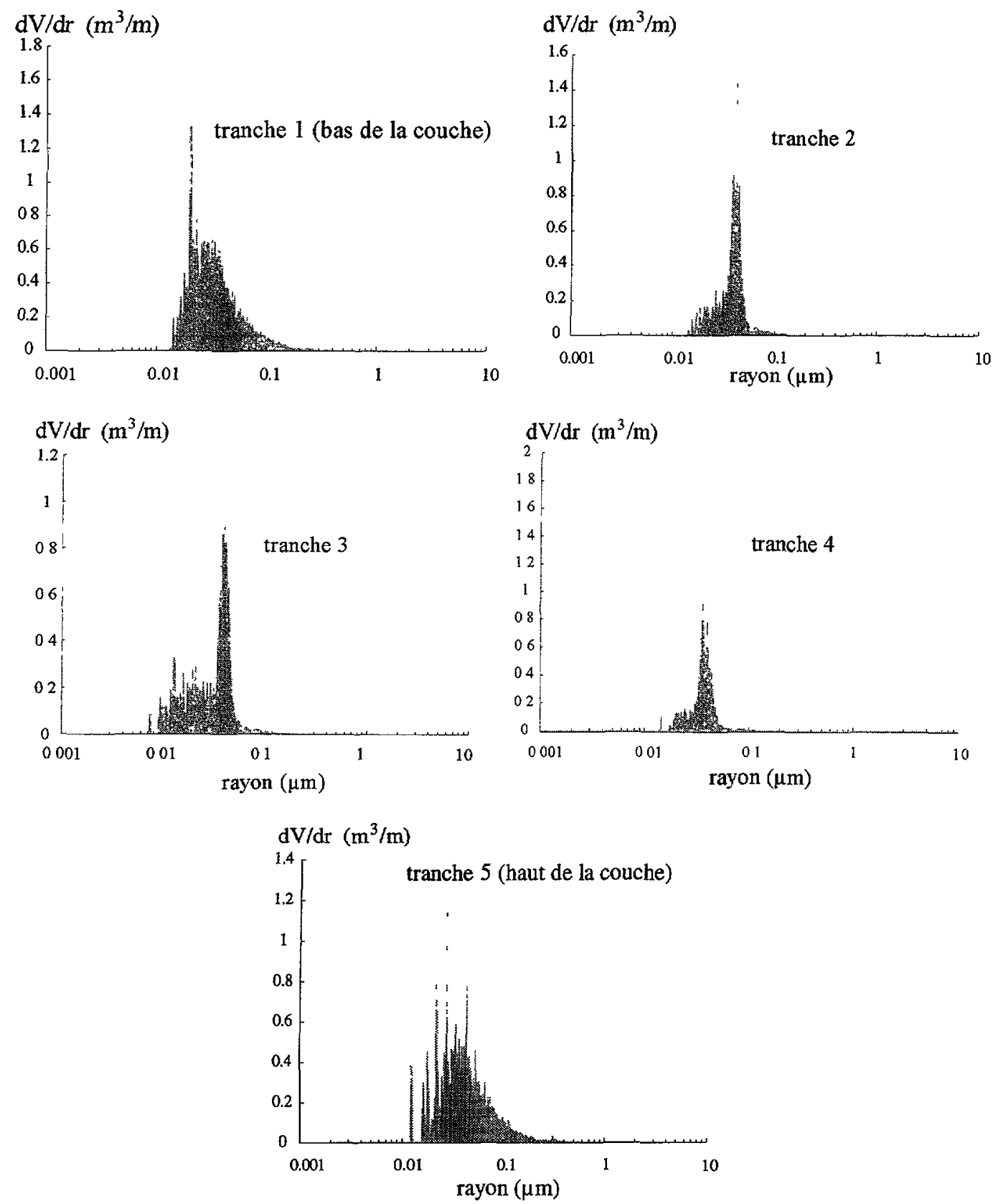

Fig. 21. - Répartition de la taille des pores dans une couche séchée à $95^{\circ} \mathrm{C}$ sans simulation d'imbibition.

[Pore size distribution in a paper coating; a $95{ }^{\circ} \mathrm{C}$ drying experiment without any impregnation simulation.] 

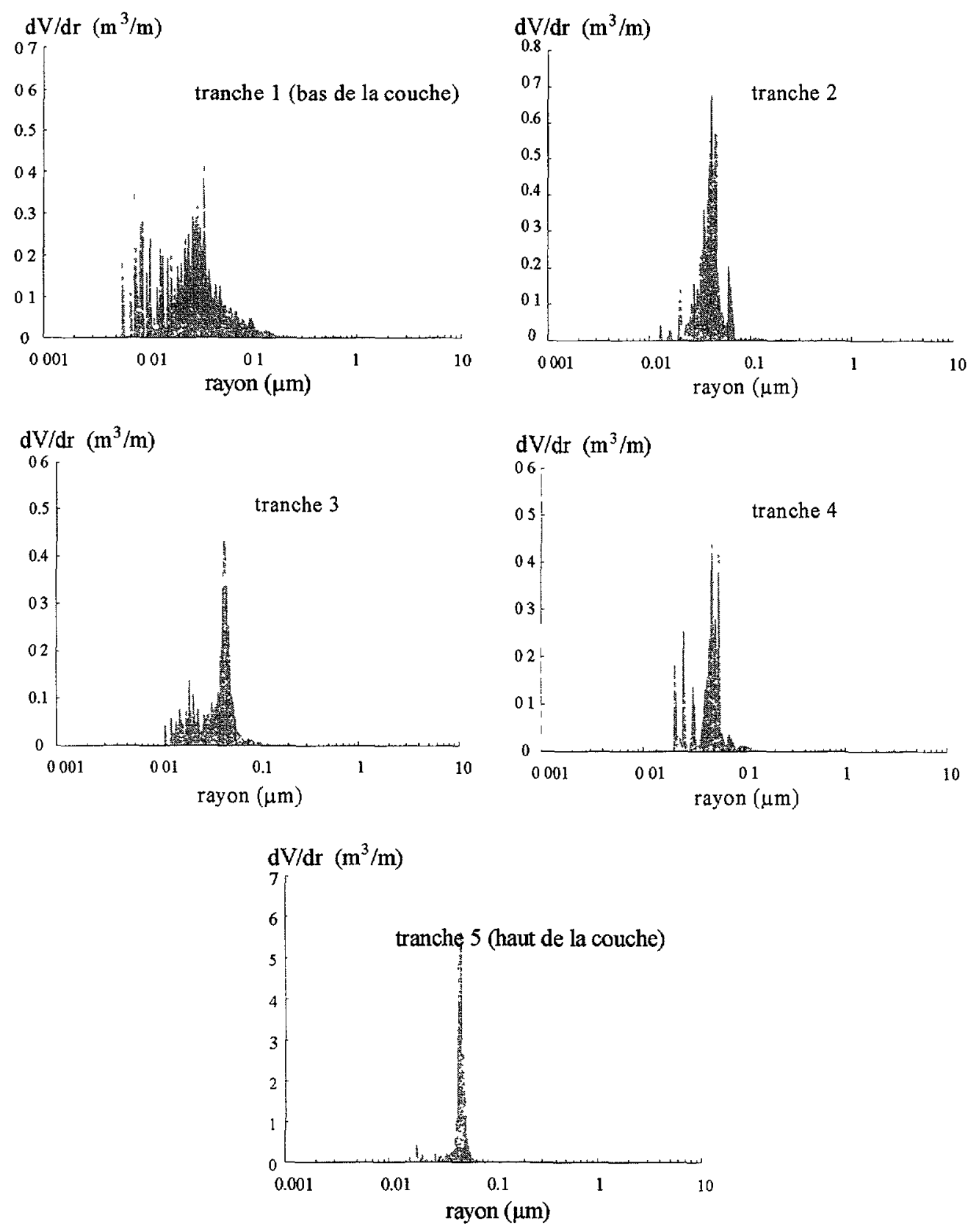

Fig. 22. - Répartition de la taille des pores dans une couche séchée à $70^{\circ} \mathrm{C}$ avec simulation d'imbibition.

[Pore size distribution in a paper coating; a $70^{\circ} \mathrm{C}$ drying experiment with our impregnation simulation.] 

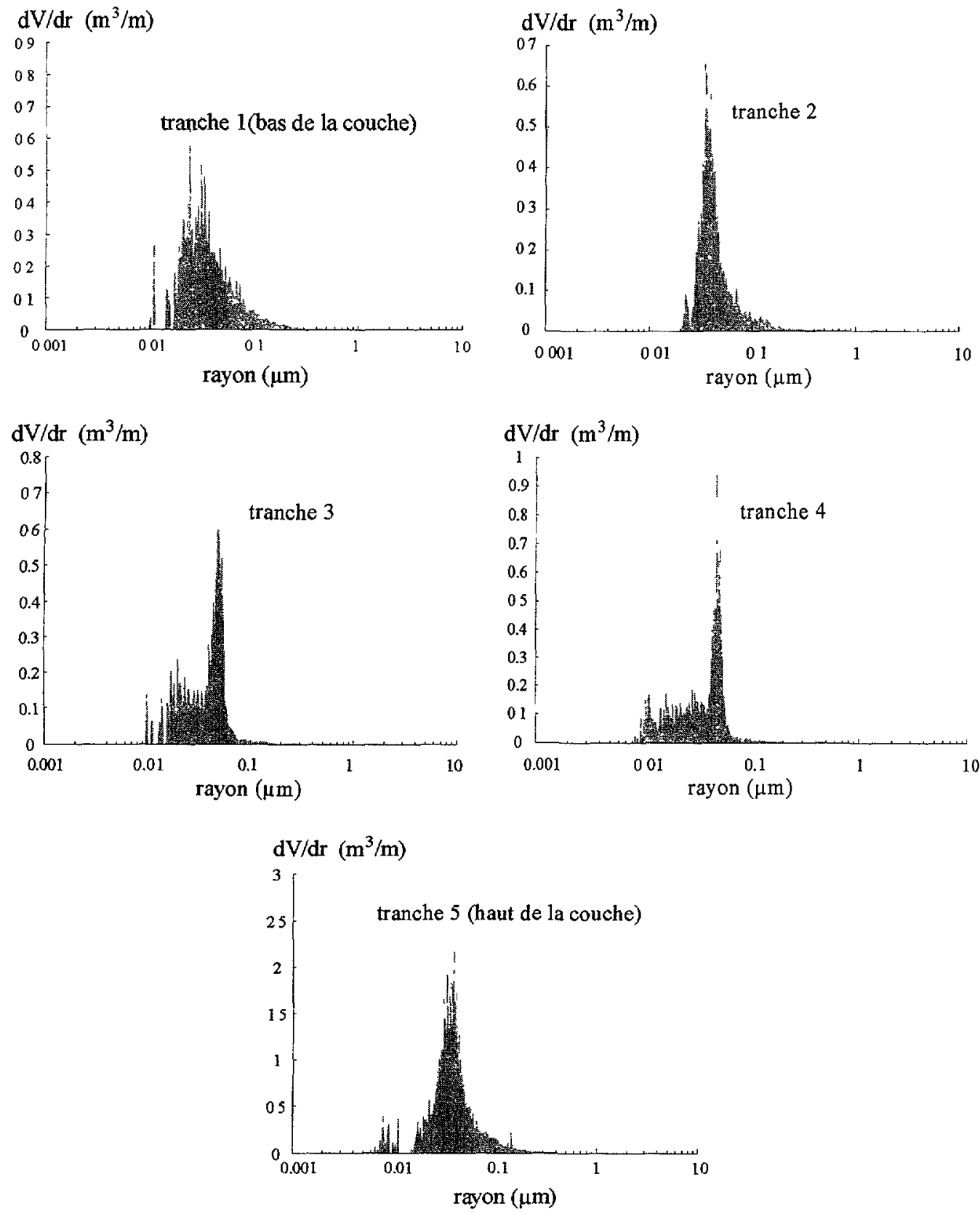

Fig. 23. - Répartition de la taille des pores dans une couche séchée à $95^{\circ} \mathrm{C}$ avec simulation d'imbibition.

[Pore size distribution in a paper coating; a $95^{\circ} \mathrm{C}$ drying experiment with our impregnation simulation.] 


\section{Bibliographie}

[1] Hagen K.G., Effect of drying; binder migration in paper and paperboard coatings, Tappi Press, Atlanta, Eds. (1993) pp. 39-59.

[2] Nadeau J. P. et Puiggali J. R., Séchage : des processus physiques aux procédés industriels (Lavoisier, Tech. et Doc., Paris, 1995).

[3] Lepoutre P. and Watanabe J., Mechanism for the consolidation of the structure of claylatex coatings, J. Appl. Polymer Sci. 27 (1982) pp. 4207-4219.

[4] Vanderhoff J. W. and Bradford E. B., The simulation of binder migration in clay-latex coatings using a model system, Proceeding of the Tappi Coating Conference 1972 (San Francisco, 1972) pp. 173-200.

[5] Lee D. I. and Whalen-Shaw M., Fundamentals and strategies; binder migration in paper and paperboard coatings, Tappi Press, Atlanta, Ed. (1993) pp. 19-38.

[6] Fujiwara H., Fujisaki N., Shimizu I. and Kano I., The effect of water penetration on offset mottling, Tappi J. (May 1989) pp. 91-97.

[7] Farkas I., Lampinen M. J. and Ojala K., Water flow and binder migration during drying of coated paper, Drying Technology 9 (1991) 1019-1049.

[8] Hagen K.G., Using infrared radiations to dry coatings, Tappi J. (May 1989) 77-83.

[9] Engström G., Ström G. and Norrdahl Ph., Studies of the drying process and its effect on binder migration and offset mottle, Tappi $J$. (December 1987) 45-49.

[10] Engström G., Rigdahl M., Kline J. and Ahlroos J., Binder distribution and mass distribution of the coating layer -cause and consequence-, Tappi J. (May 1991) 171-179.

[11] Nowicki S. C., Davis H. T. and Scriven L. E., Computer-aided modeling of binder migration; binder migration in paper and paperboard coatings, Tappi Press, Atlanta, Ed. (1993) pp. 1-18.

[12] Vanderhoff J. W. and Bradford E. B., The simulation of binder migration in clay-latex coatings using a model system, Proceeding of the Tappi Coating Conference (San Francisco, 1972) pp. 173-200.

[13] Moyne C., Contribution à l'étude du transfert simultané de chaleur et de masse au cours du séchage sous vide d'un bois résineux, Thèse de l'Institut National Polytechnique de Lorraine (Nancy, 1982).

[14] Couture F., Modélisation fine d'un problème de séchage : développement d'outils adaptés, Thèse de l'Université Bordeaux I (1995).

[15] Mailly-Lejeune V, Contribution à l'étude de la permanence des propriétés optiques des papiers couchés, Thèse de l'T.N.P. de Grenoble (1994).

[16] Moreau S., Structures des couches pigmentaires, étude de leur séchage, Thèse de l'I.N.P. de Grenoble (1994).

[17] Bernada P. and Bruneau D., Modeling binder migration during drying of a paper coating, Soumis à TAPPI $J$. en septembre 1995.

[18] Takase K., Miura H., Tamon H. and Okazaki M., Structure formation of coated films with dispersed pigments during drying, Drying technol. 6 (1994) 1279-1296. 